Revue des patrimoines

$40 \mid 2019$

Lits historiques. Première anthologie des lits européens du XVe au XIXe siècle

\title{
Das Bett des ersten Schlafzimmers König Ludwigs II. im Schloss Linderhof
}

\section{Sybe Wartena}

\section{(2) OpenEdition}

Journals

Édition électronique

URL : http://journals.openedition.org/insitu/24048

DOI : $10.4000 /$ insitu. 24048

ISSN : 1630-7305

Éditeur

Ministère de la Culture

Référence électronique

Sybe Wartena, « Das Bett des ersten Schlafzimmers König Ludwigs II. im Schloss Linderhof », In Situ

[En ligne], 40 | 2019, mis en ligne le 19 septembre 2019, consulté le 04 octobre 2019. URL : http://

journals.openedition.org/insitu/24048 ; DOI : 10.4000/insitu.24048

Ce document a été généré automatiquement le 4 octobre 2019.

\section{(c)}

In Situ Revues des patrimoines est mis à disposition selon les termes de la licence Creative Commons Attribution - Pas d'Utilisation Commerciale - Pas de Modification 4.0 International. 


\title{
Das Bett des ersten Schlafzimmers König Ludwigs II. im Schloss Linderhof
}

\author{
Sybe Wartena
}

\section{Die absolutistischen Bauprojekte Ludwigs II. und die besondere Rolle der Schlafzimmer}

1 Ludwig II. begann sich 1868 mit einem Bauvorhaben beim Jagdhaus seines Vaters beim Linderhof im Graswangtal in den Allgäuer Alpen zu beschäftigen ${ }^{1}$. Dieses Gebäude sollte Versailles zitieren, das Ludwig II. 1867 kennengelernt hatte, zunächst in extremer Abbreviatur. Als Codename für das Bauprojekt wählte Ludwig II. ein Anagramm des Mottos König Ludwigs XIV. von Frankreich, l'Etat, c'est moi: Tmeicos Ettal, womit auch auf das nahe gelegene Kloster Ettal verwiesen war. Mehrfache Planerweiterungen führten dazu, dass eine Verwirklichung im Graswangtal nicht mehr möglich war und dass der König im September 1873 die „Herreninsel“ im Chiemsee kaufte, wo ausreichend Platz zur Verfügung stand und der Bau 1878 begonnen wurde. Mit weiteren Planänderungen - bis 1883 sind 18 aufeinander aufbauende Stadien nachweisbar - wurde letztendlich eine exakte Kopie von Versailles angestrebt.

In Linderhof plante der König parallel ein zweites Gebäude, das ebenfalls mehrfach vergrößert wurde und das, wie das eigentliche Versailles-Projekt, eine Kulisse für Idealbilder des französischen Absolutismus werden sollte. Hier ist erstmals am 30. September 1870 der Plan für einen kleinen Anbau von drei Räumen an das „Königshäuschen“, das Jagdhaus Maximilians II. dokumentiert. Kaum war dieser Anbau aus einem ovalen und zwei kleinen hufeisenförmigen Räumen vollendet, wurde ein entsprechender, parallel angeordneter und ein diese beiden Trakte im Norden verbindendes Schlafzimmer geplant. Damit entstand eine kleine Dreiflügelanlage, geschlossen von dem alten, ganz anders gearteten Jagdhaus als viertem Flügel im Süden (Abb. 1). Die Ausstattung der drei neuen Flügel im Stil des Neo-Rokoko war Ende 
1872 abgeschlossen, doch wurde schon 1873 das Schlafzimmer vergrößert und verändert.

Abb. 1

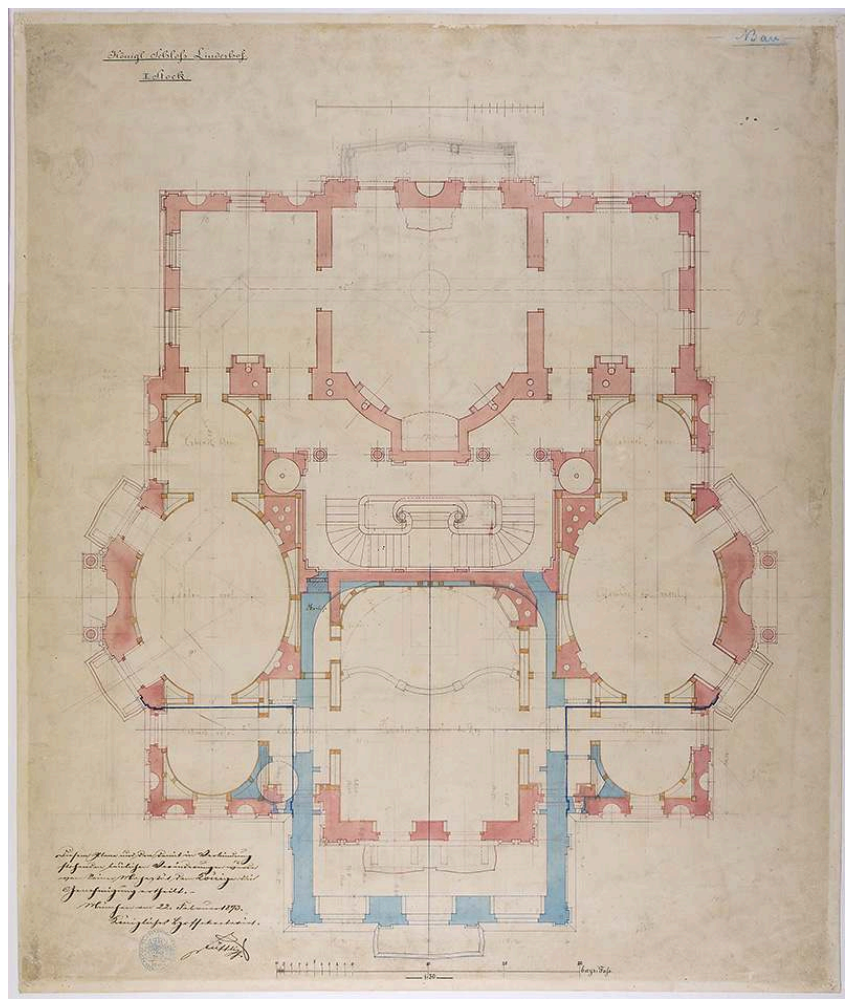

Grundriss Schloss Linderhof, Anfang 1873. Der Plan ist nach Süden gerichtet. Der nördliche (untere) Teil zeigt den damaligen Bauzustand, der südliche die projektierte Vollendung anstelle des Königshäuschens (genehmigt 22.2.1873). Zusätzlich ist die 1884 angeordnete Vergrößerung des Schlafzimmers eingetragen. BSV. L.II.-Mus. 1958.

(c) Bayerische Schlösserverwaltung. www.schloesser.bayern.de.

1873 begann auch die konkrete Planung für das Ersetzen des alten „Königshäuschens“ durch einen neuen Süd-Trakt, der zugleich die Schauseite des Schlösschens bilden sollte. Eine wohl schon 1872 entstandene, nicht ausgeführte Planungsvariante sah vor, als Südriegel eine "Chambre de Parade“ zwischen einem „Salon de l'Oeil de Boeuf" und einer "Salle de la Garde“ zu schaffen ${ }^{2}$ - der Gedanke an Versailles war also auch für Linderhof eine der Inspirationsquellen. Wie später in Herrenchiemsee war die größere "Chambre de Parade“ wohl nicht zur praktischen Nutzung gedacht sondern als Denkmal für Ludwig XIV., während Ludwig II. selbst in dem kleineren RokokoSchlafzimmer schlafen wollte. ${ }^{3}$ Anstelle dieses Plans wurde jedoch als zentraler Raum der Südseite als Gegenstück zum nördlichen Schlafzimmer der Spiegelsaal in Anlehnung an das Spiegelkabinett der Reichen Zimmer in der Münchner Residenz gebaut. $^{4}$

4 Nach dem Abschluss der Ausstattung des südlichen Traktes 1877 blieb Schloss Linderhof zunächst unverändert und die Ressourcen wurden ganz auf den großen Bau auf der Insel im Chiemsee konzentriert, dessen Ausführung 1878 begann. Dort entstanden zwei Appartements mit Schlafzimmern, die an Ludwig XIV. und Ludwig XV. von Frankreich erinnern sollten, letzteres zum Bewohnen durch Ludwig II. gedacht. Für das Bett in der Ludwig XIV. gewidmeten Chambre de Parade wurden die ersten 
Ausführungspläne 1876 angefertigt, die letzten Arbeiten waren 1883 abgeschlossen. Im selben Jahr folgten erste Entwürfe für das Bett im kleinen Appartement Ludwigs XV., das 1886 vollendet wurde. In Linderhof ordnete der König 1884 an, das Schlafzimmer zu vergrößern und komplett neu einzurichten. Die Vollendung dieses Projekts erlebte er nicht mehr. Nach seinem Tod am 13. August 1886 wurden die Schlösser Ludwigs II. zur Besichtigung geöffnet und das Linderhofer Schlafzimmer den Besuchern zunächst im Rohbau gezeigt. ${ }^{5}$ Dann wurden die fertiggestellten Ausstattungsstücke und teilweise die alten Möbel wieder eingebracht. Teile der alten Einrichtung, die nicht mehr gebraucht wurden, wurden 1887 vom Hofsekretariat dem Bayerischen Nationalmuseum übertragen. 1968 gelangten sie als Dauerleihgabe an die Bayerische Schlösserverwaltung, die sie im Ludwig II.-Museum im Schloss Herrenchiemsee ausstellt.

\section{König Ludwigs Rekonstruktionsversuche des Bettes Ludwigs XIV. in Versailles}

Die Beschäftigung Ludwigs II. mit den Schlafzimmern der Französischen Könige in Versailles hatte schon vor den Bauprojekten in ländlicher Abgeschiedenheit begonnen. In Zusammenhang mit der Neueinrichtung seines Appartements in der Münchner Residenz 1867 verlangte Ludwig nach dem „... prunkvollen, erhabenen Styl, wie er zur Zeit Ludwigs XIV. der herrschende war, und der bei Herstellung meiner Gemächer der einzig maßgebende sein soll...".6 Der Entwurf des Schlafzimmers griff verschiedene Punkte der Chambre de Parade von Versailles auf, wie sie seit der Einrichtung unter Louis Philippe 1837 präsentiert wurde: eine Balustrade, ein axial stehendes Baldachinbett in ähnlichen Formen, das Bett flankierende Gemälde, eine Büste des Sonnenkönigs. ${ }^{7}$ Diese Elemente wurden durch Bildquellen vermittelt, nach denen Ludwig immer wieder seine Agenten recherchieren ließ. In seinem Besitz waren nicht nur die programmatische Monographie zu Versailles von Jean Vatout, Band 1 der Reihe Souvernirs historiques des Résidences Royales de France von 1837 und das großformatige Tafelwerk Souvenir d'une Promenade à Versailles von 1838, sondern auch eine Fotografie der Chambre de Parade, die noch weitgehend dieselbe Situation zeigte. ${ }^{8}$ Diese Ansichten scheinen die Basis für die von Ludwig beauftragten Künstler gewesen zu sein, denn unter dem Entwurfs- und Planmaterial seiner Bauprojekte, das vom Wittelsbacher Ausgleichsfonds bzw. von der Bayerischen Schlösserverwaltung verwahrt wird, finden sich ein Aquarell, das die Fotografie kopiert und zwei Einzeldarstellungen des Bettes und seiner direkten Umgebung, die dessen Grundform exakt wiedergeben, die Gliederung und Komposition sowie Ikonografie der Behangteile aber "korrigieren" (Abb. 2). 


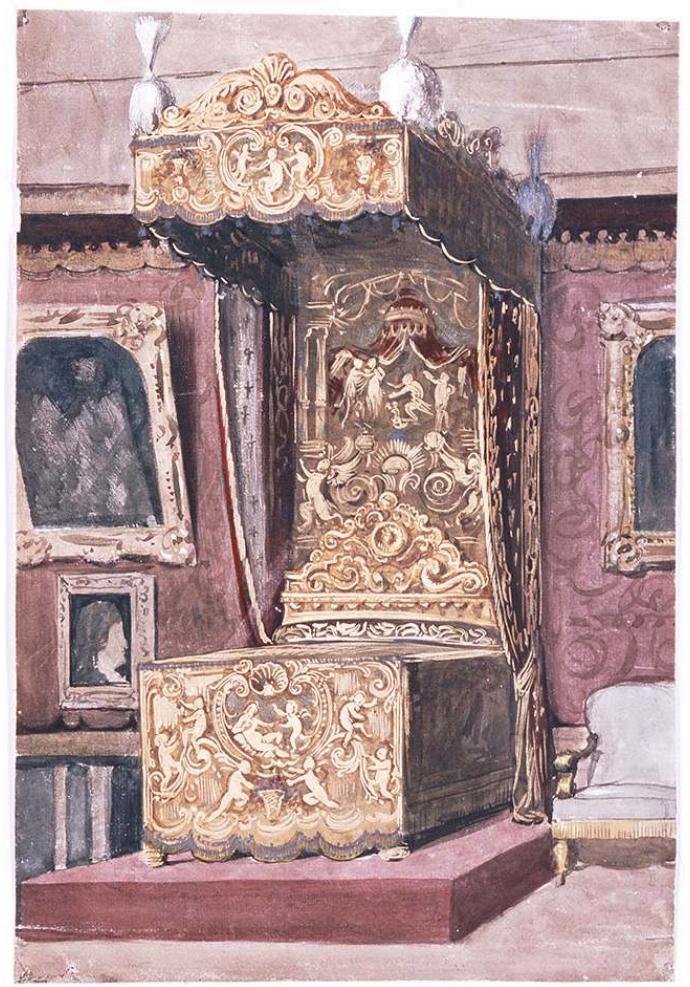

Erster Rekonstruktionsversuch des Bettes Ludwigs XIV. von Simon Delobel, dargestellt in der Chambre de Parade von Versailles im Zustand nach 1837. Welcher der von Ludwig II. beauftragten Künstler das Blatt zwischen 1867 und 1869 schuf, ist unbekannt. Gouache mit Gold, 36 × 24,5 cm, WAF Inv.-Nr. B VIII 47.

(c) WITTELSBACHER AUSgLEICHSFONDS MÜNCHEN.

Denn Ludwig II. wollte offenbar das Bett rekonstruieren, das Ludwig XIV. von 1682 bis 1701 im späteren Salon de Mercure benutzt hatte und dessen zeitgenössische Beschreibung durch einen Marquis de Soucy bei Vatout publiziert war. Es wurde als Gipfel der Textilkunst gepriesen. Der Hoftapissier Simon Delobel soll zwölf Jahre daran gearbeitet haben. Ludwig II. muss von der Beschreibung sehr beeindruckt gewesen sein, denn er ließ sie ins Deutsche übersetzen. ${ }^{9}$ Ob Ludwig II. erkannt hatte, dass das 1837 in der Chambre de Parade aufgestellte und heute im Salon de Mercure stehende Bett entgegen der Behauptung bei Vatout mit dem Bett Ludwigs XIV. nichts zu tun hatte, oder ob er im Streben nach Idealen Formen die Abweichung vom für original gehaltenen Bett in Kauf nahm, ist ungewiss. ${ }^{10}$ Alle Entwurfsstadien des Bettes für die Chambre de Parade des Versaillesprojekts griffen die Ikonographie des Delobel-Bettes Ludwigs XIV. auf. Sie zeigen zahlreiche Amoretten und Venusdarstellungen und als Hauptbild an der Bettrückwand die Flügelbeschneidung Amors durch Nymphen unter einem Baldachin in prächtiger architektonischer Rahmung.

7 Die Formfindung der Betten für Linderhof und für die Chambre de Parade von Tmeicos Ettal läuft zu Beginn synchron und beide scheinen dieselbe Wurzel zu haben. Nach diesem schon erwähnten frühesten Versuch, der die Form des Versailler Bettes von 1837 beibehielt und nur den Dekor „rekonstruierte“, folgte wahrscheinlich 1869 der erste eindeutig Tmeicos Ettal zuzuordnende Bettentwurf aus einem Satz reich ausstaffierter Blätter, welche die Beschreibung De Soucys exakt umsetzen - ein erster Entwurf mit vier Stützen, gefolgt von zwei Ansichten im Typus „à la duchesse“ mit 
Stützen nur am Kopfende. ${ }^{11}$ Hier hat das Bett gerade Formen, nur der Baldachin ist durch eine Kuppel und Lambrequins mit mehreren dreipassförmigen Ausbuchtungen und Quasten bewegt. Die Pfosten, deren Formensprache ans Louis XVI. erinnert, sollten reich gedrechselt, geschnitzt und vergoldet werden, doch den reichsten Schmuck sollten die Textilien aufweisen, die stilistisch näher am Spätbarock stehen: die Entwürfe für Bettrückwand, Couvertdecke mit seitlichen Behängen, Lambrequin und Bonnes Grâces sind mit goldenem Fond, goldenen, silbernen und wenigen polychromen Reliefstickereien aufs reichste gestaltet. Die Vorhänge weisen entsprechende Stickereien auf rotem Fond auf. ${ }^{12}$

\section{Von der rechteckigen zu einer bewegten Form}

1872 zeigt ein Entwurf für Tmeicos Ettal ein von Versailles deutlich abweichendes, vergrößertes Schlafzimmer. Auch Bett und Baldachin wurden überarbeitet: Statt rechteckiger Formen sind sie jetzt in mehreren Kurven geschweift, die oberen und unteren Konturlinien beider Körper stark bewegt und die Vorderkanten des Bettes als weibliche Karyatiden gestaltet und S-förmig geschweift. ${ }^{13}$

Abb. 3

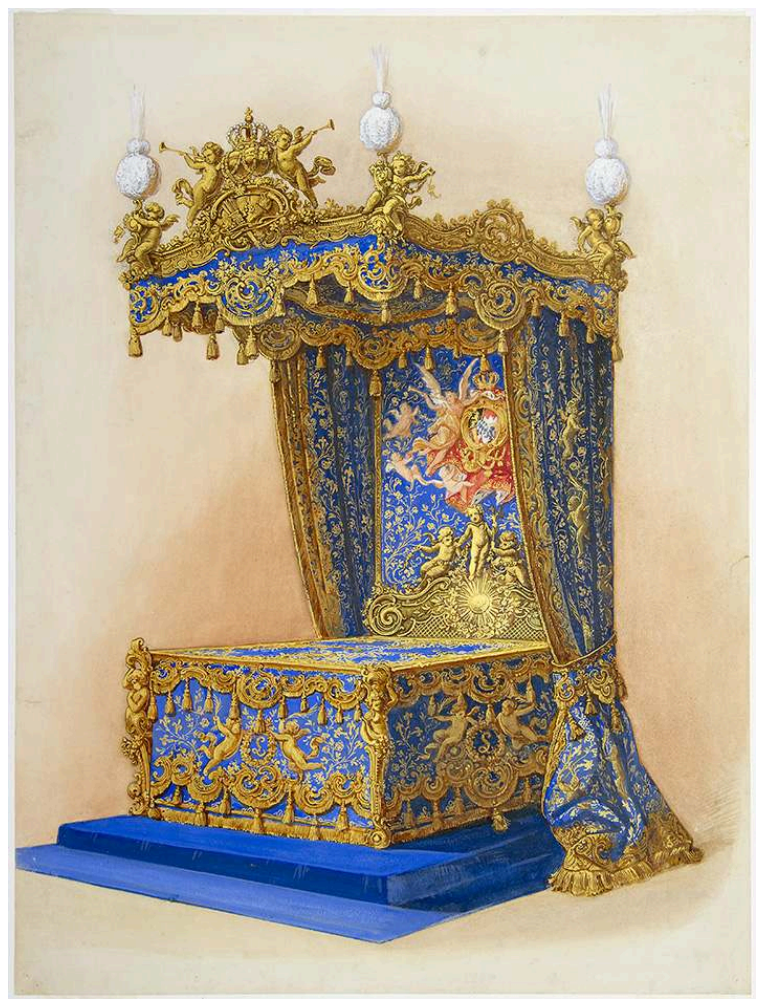

ERSTER ENTWURF FÜR DAS BETT DES ERSTEN LINDERHOFER SCHLAFZIMMERS, FRANZ SEITZ 1871. GOUACHE UND GOLD, 57 X 43 CM. BSV, L.II.-MUS. 2522A.

(c) Bayerische Schlösserverwaltung. www.schloesser.bayern.de.

9 Für Linderhof sind um dieselbe Zeit ähnliche Entwicklungen zu beobachten. Der erste überlieferte Entwurf für das Bett ist durch ein mit Gouache und Gold reich ausgeführtes Blatt dokumentiert (Abb. 3), das mit einer Zahlung von 88,- fl. an Franz Seitz im Jahr 1871 für die „Zeichnung“ eines „blauen Himmelbettes“14 in Zusammenhang 
gebracht wird. Das Bett selbst weist rechtwinklige Formen auf, die vorderen Kanten sind jedoch, wie das Tmeicos-Ettal-Bett von 1872, von leicht S-förmig geschweiften, geschnitzten Karyatiden gebildet - links einer männlichen und rechts einer weiblichen mit Füllhorn im Arm. Die Behänge der auf einem Rahmen montierten Couvertdecke ${ }^{15}$ schließen eng an diese Ecklösung an. Der Baldachin ist im Grundriss geschweift und auch in der oberen und unteren Konturlinie stark bewegt. Die obere Leiste ist geschnitzt, die Textilien mit schweren Reliefstickereien ausschließlich in Gold auf blauem Grund auszuführen gedacht. Von der Liebesthematik der Entwürfe für Tmeicos Ettal ist nur ein einziges Motiv direkt übernommen worden: die Waffen Amors in der zentralen Kartusche am Baldachin (Köcher, Bogen und Fackel), die am Bett Ludwigs XIV. nach der Beschreibung an zwei Stellen auftraten. ${ }^{16}$ Darüber präsentieren zwei Trompete blasende Putti die Bayerische Königskrone mit Ordensketten auf einem Kissen. An den Behängen halten je zwei Putti die Initiale des Königs - beziehungsweise Ludwigs XIV. oder XV. - in einem Blütenkranz, weitere gestickte Amoretten bevölkern zwischen Ranken flatternd die Außenseiten oder äußeren Vorhänge. Die inneren Vorhänge weisen lediglich goldene Ranken auf. ${ }^{17}$ Die Bettrückwand zeigt als Hauptmotiv das Bayerische Wappen auf einem roten Wappenmantel, getragen von zwei abermals Posaune blasenden Genien. Der geschnitzte Aufsatz des Kopfendes kann als geschweifter Giebel beschrieben werden, in dessen Zentrum die Sonne strahlt - ein Motiv des 1837 in Versailles installierten Bettes, das bei De Soucy nicht beschrieben war und dennoch von Ludwig II. am Bett in der Residenz und an allen vier Betten für Herrenchiemsee und Linderhof übernommen wurde. In Linderhof sind auf dem Aufsatz zwei Putti mit Blumengirlanden angeordnet, die einen erhöht stehenden mit einer Lilie flankieren.

10 Als Grundidee der Gliederung wie auch der ornamentalen Gestaltung des Bettes können Friese aus verketteten C-Bögen gelten, welche die untere wie die obere Zone der Behänge der Couvertdecke an Längsseiten und Fußende sowie Ober- und Unterkante des Baldachins akzentuieren. Dabei ist die Baldachin-Oberkante geschnitzt und jede der drei Seiten hat mittig einen bogenförmigen Auszug, während die übrigen drei Friese hängende Lambrequins darstellen. Die hängenden C-Bögen mit Akathus- und Rocaillenbesatz sollten in Reliefstickerei ausgeführt, unten jeweils von einem dichten Goldfransenbesatz begleitet und mit zahlreichen hängenden Goldquasten garniert werden. 


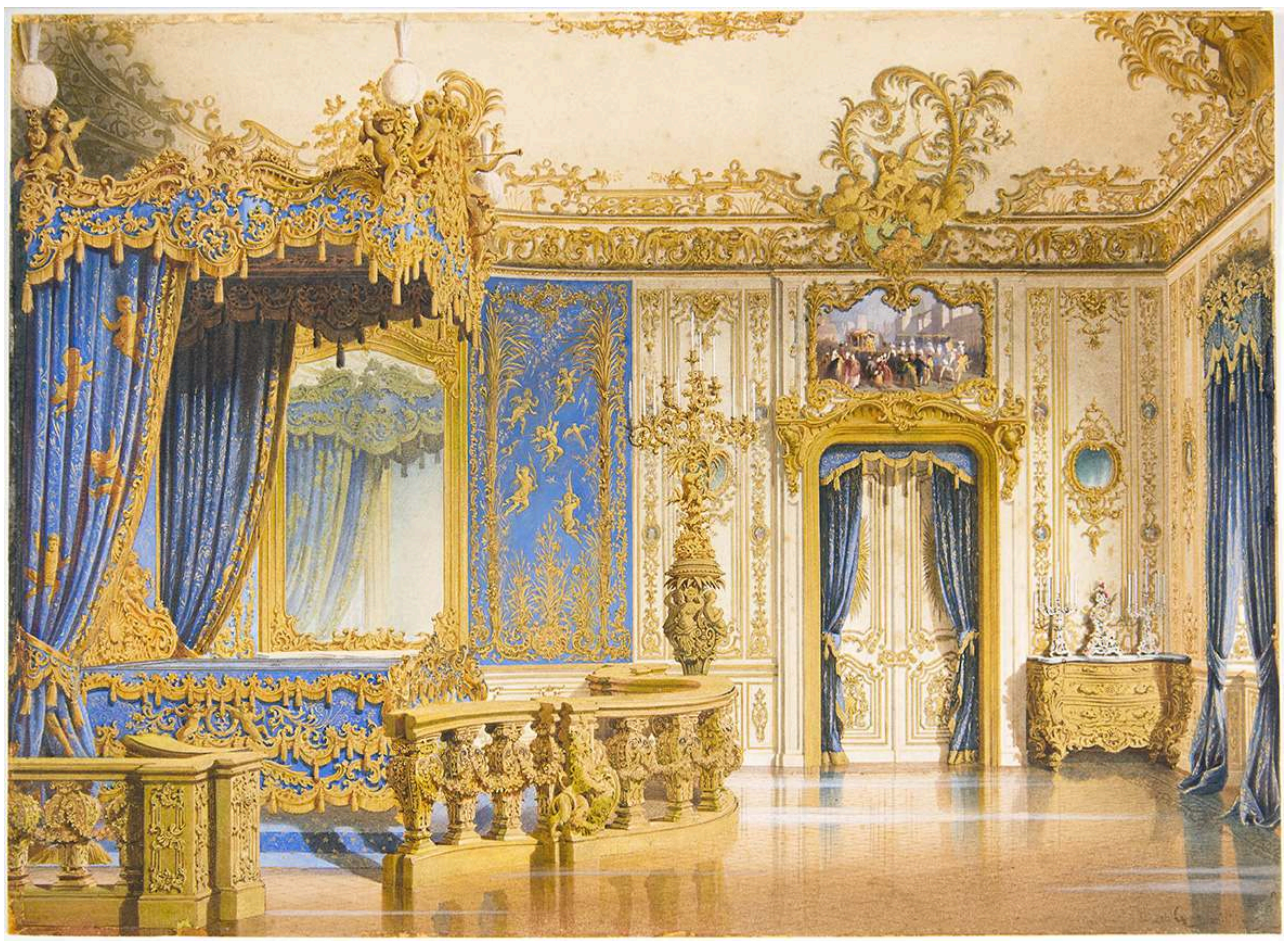

VISIERUNG DES ERSTEN SCHLAFZIMMERS VON SCHLOSS LINDERHOF, SIGNIERT UND DATIERT VON ANgElo QUAgLIO, 1871. AQUARELL UND GOLD, 39 x 53,5 CM. BSV, L.II.-MUS. 265.

(C) Bayerische Schlösserverwaltung. www.schloesser.bayern.de.

11 Im selben Jahr 1871 schuf Angelo II. Quaglio eine Raumansicht (datiert und signiert), die das Bett in etwas abweichender Form zeigt (Abb. 4). ${ }^{18} \mathrm{Zu}$ diesem Stadium liegt auch eine Visierung des Bettes alleine vor, die aufgrund einer Zahlung von 90,- fl. Im Jahr 1871 für die „Zeichnung einer Bettlade mit Himmel“" ${ }^{19}$ schon lange Adolph Seder zugeschrieben ${ }^{20}$ aber bisher nicht publiziert wurde (Abb. 5). Erst jetzt wurde die offenkundig zeitgenössische Aufschrift beachtet: „Neue Bettzeichnung von Seder. Baldachin und Bettgestell (vorn) geschweift. Auf den Tritt sollen Sterne gestickt werden." Der wesentliche Unterschied zum vorangehenden Entwurf ist, dass das Fußende jetzt über die auf einen Rahmen montierte und zwischen die Betthäupter hoch über der Matratze eingehängte Couvertdecke hinaussteht. Dadurch hat diese nur noch seitlich Behänge, nicht mehr am Fußende. Das Fußende besteht nun aus einem annähernd ovalen Rahmen und einer textilen Füllung und unterbricht die im früheren Entwurf durch die umlaufenden Bogenfriese gegebene waagerechte Gesamtgliederung. Das Fußende dominiert nun das ganze Bett, wodurch dessen Charakter vom reinen Textilmöbel zum Bildhauermöbel verschoben wird. Zugleich wird der Baldachin stärker bewegt, sowohl im Grundriss als auch besonders in der oberen Konturlinie, die jetzt nur noch aus Bögen besteht, während sie im vorhergehenden Entwurf auch leicht geschwungene sowie gerade Partien enthielt. Die Gliederung mit waagerechten Bogenfriesen bleibt gültig, verliert aber durch die Unterbrechung an der Front ihre Dominanz. 
Abb 5

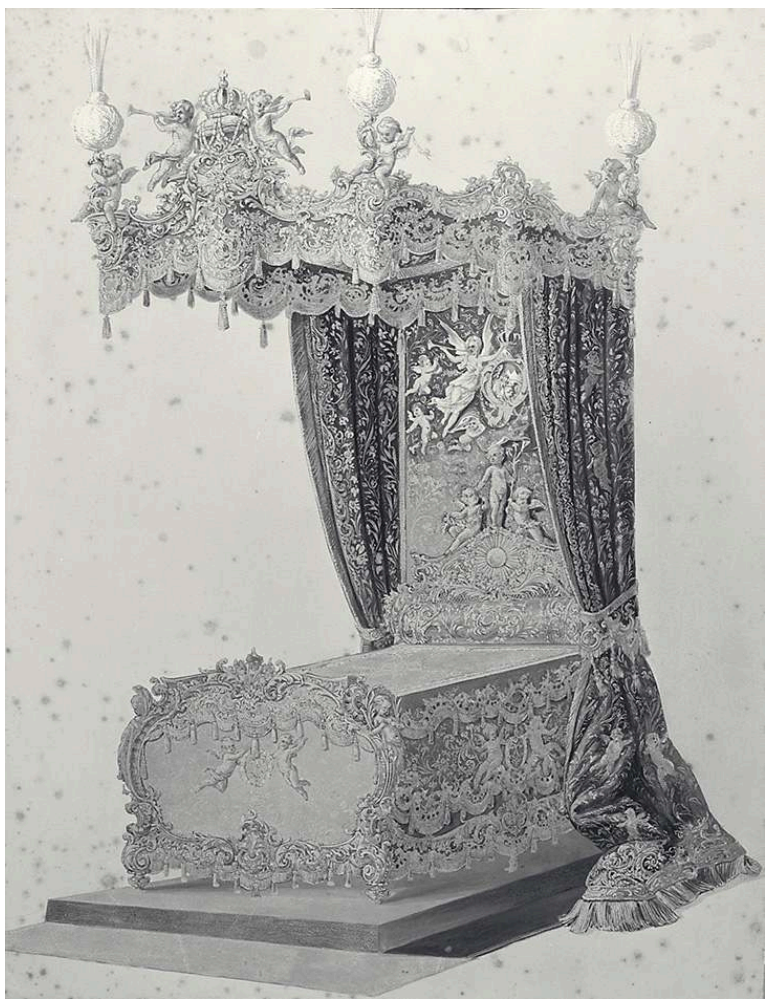

Zweiter Entwurf für das Bett des ersten Linderhofer Schlafzimmers, Adolph Seder, 1871, Gouache mit Gold, 59 × 45,5 cm. WAF Inv.-Nr. B VIII 39.

(c) WITTELSBACHER AUSgLEICHSFONDS MÜNCHEN.

Die Abweichungen vom Seder-Entwurf zur Ausführung - "Schreiner- und OrnamentenBildhauer-Arbeiten" von der Firma Anton Pössenbacher, figürliche Bildhauerarbeiten von Johann Hirt, Vergoldung Firma Radspieler, Stickereien Atelier Mathilde Jörres, Firmen Alckens und Bornhauser, Zahlungen 1872, Restzahlungen $1873^{21}$ - halten sich in so engen Grenzen, dass keine weiteren Entwurfsstadien anzunehmen sind (Abb. 6). Die geringen Veränderungen können mit der Umsetzung der perspektivischen Gesamtansicht in einzelne Werkszeichnungen erklärt werden. Durchgängig fällt nur der Ersatz der Rocaillenbögen, welche den Kontur der Lambrequins bilden, durch akanthusartige Blattwedel auf - die Rocaillenbögen finden sich in der Ausführung nur vereinzelt am Fußende und an den vorderen und unteren Bordüren der großen Vorhänge sowie den Bordüren der Wandpanneaux. 
Abb. 6

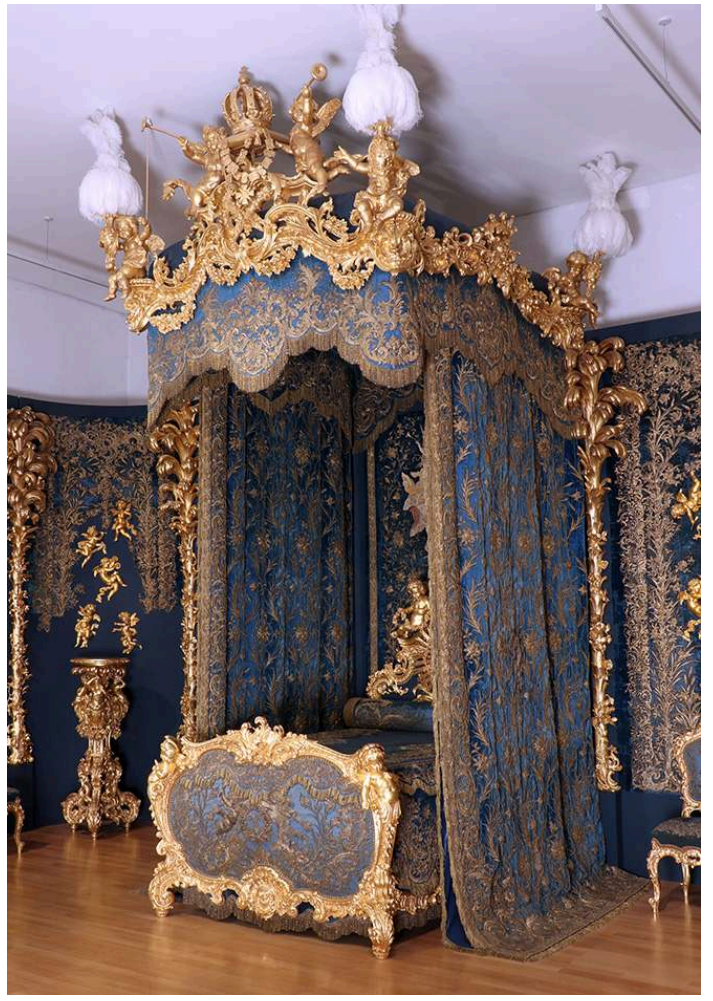

Gesamtansicht des Bettes, Zustand 2018. Foto Sybe Wartena.

(c) Bayerisches Nationalmuseum.

Die Akzentverschiebung, die in Linderhof 1871 auftrat, kann mit dem spätestens 1872 erfolgten Wechsel vom rechtwinkligen Stollenbett zum bildhauerisch geprägten Bett für Tmeicos Ettal verglichen werden. Die Frage nach der zeitlichen Abfolge kann mangels weiterer datierter Quellen jedoch nicht eindeutig beantwortet werden. ${ }^{22}$ Das gilt genauso für die zweite Planänderung, die sich in beiden Projekten um dieselbe Zeit vollzog: neu hinzugefügte raumhohe, geschnitzte und vergoldete Palmen als Trennglieder zwischen den Wandbespannungsfeldern der Bettnische. Eine Wandabwicklung für Linderhof ${ }^{23}$ trägt folgende Korrekturangabe vom 12.08.1872 von Lorenz Düfflipp, dem Hofsekretär, über den Ludwig meist mit seinen Künstlern kommunizierte: „Rückwand des Schlafzimmers soll ganz mit blauem Samt überzogen werden, auch die einzelnen die Felder trennenden Streifen" - dargestellt ist zugleich die neue Gliederung durch Palmen über durchgehender Wandbespannung (Abb. 7). Bis dahin war in den Entwürfen die Trennung der textilen "Panneaux" durch weiße Lisenen mit vergoldeter Ornamentik geplant (Abb. 8). 
Abb. 7

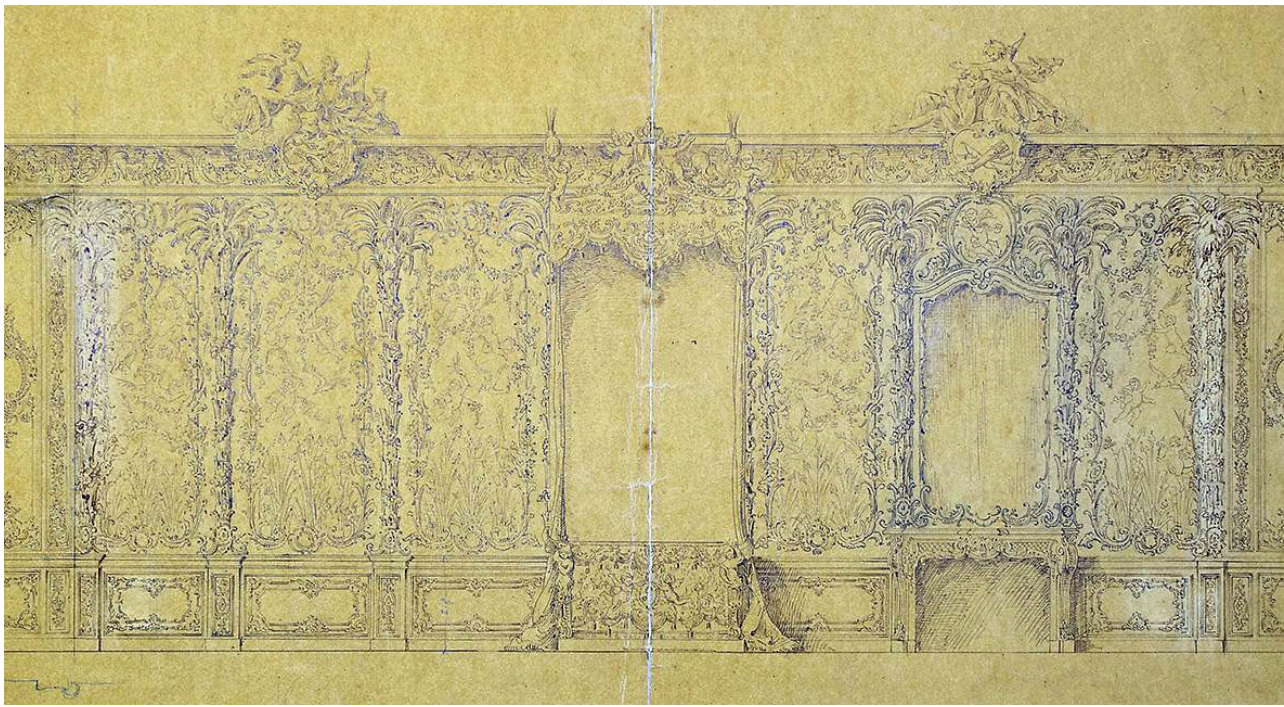

ENTWURF DES ERSTEN LINDERHOFER SCHLAFZIMMERS, WANDABWICKLUNg, GEORg DOLLMANN, 1872; PAUSE, AUSSCHNITT. BSV, L.II.-MUS. 2000/2.

(C) Bayerische Schlösserverwaltung. www.schloesser.bayern.de.

Abb. 8

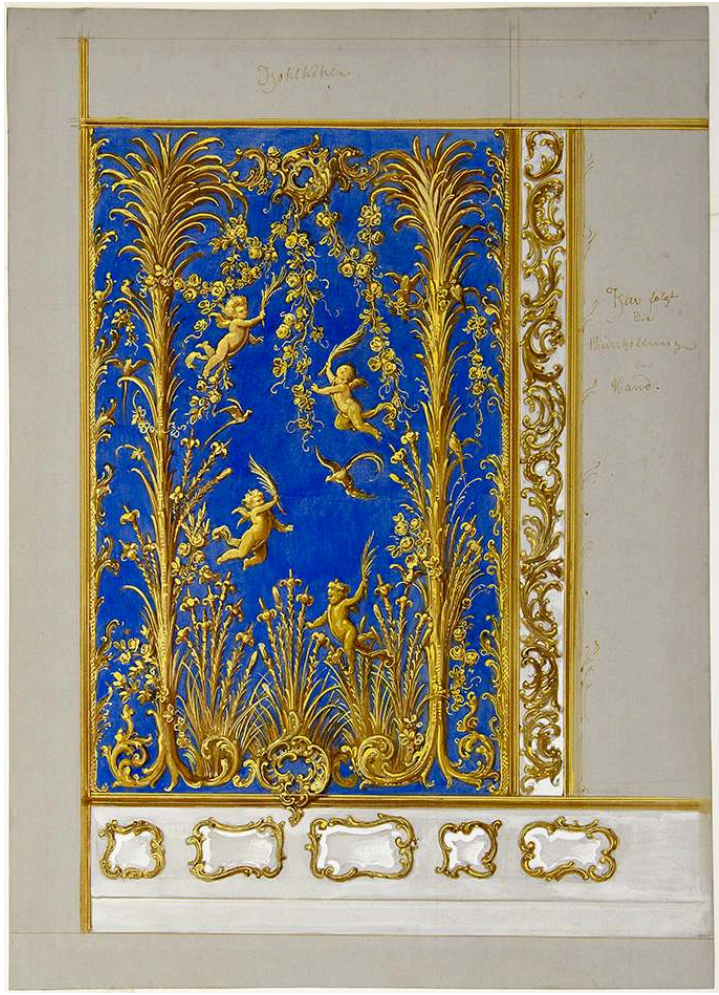

ENTWURF FÜR EIN WANDFELD DER BETTNISCHE DES ERSTEN SCHLAFZIMMERS VON SCHLOSS LINDERHOF, FRANZ SEITZ, 1871. GOUACHE MIT GOLD, 55,3 X 39,5 CM. BSV, L.II.-MUS. 2029.

(c) Bayerische Schlösserverwaltung. www.schloesser.bayern.de.

Die spätere Planung sieht neben den Palmen vor, die gestickten „Panneaux“ nicht unwesentlich zu verändern: die seitlichen, hohen „Lilienstauden“, die ihrerseits an 
Palmen erinnern, und die Kanteneinfassung, die im Entwurf als halber Blattstab angelegt war, sollten von einem feinen, mit einer Blumenranke umwundenen Stab ersetz werden. Ausgeführt wurden aber doch die „Lilienstauden“ und die schmale Randeinfassung, letztere als Blattwerkbordüre. 1872 wurde Adolph Seder für die Zeichnung einer Palme und für Änderungen in den Panneaustickereien bezahlt, ${ }^{24}$ die Ausführung der Änderung wurde 1873 abgerechnet. Diese Änderungen - Anstückungen in weniger dichter Ornamentik - sind deutlich zu erkennen (Abb. 9).

Abb. 9

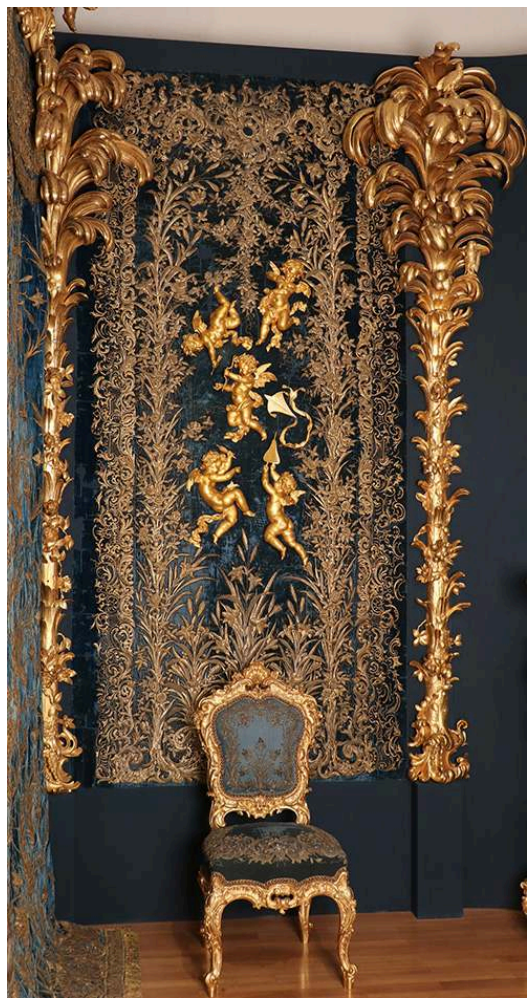

WANDFELD RECHTS DES BETTES, ZUSTAND 2018.

Foto Sybe Wartena. (c) Bayerisches Nationalmuseum.

15 Die Palmen wurden von Goertz überzeugend auf die Palmen der Logenrahmungen im Münchner Residenztheater von François Cuvilliés (um 1751-55) zurückgeführt. ${ }^{25}$ Dies könnte als Indiz dafür gewertet werden, dass das Motiv für Linderhof gefunden wurde, da die Vorbilder für das Versailles-Projekt in der höfischen Bau- und Einrichtungskunst Frankreichs, und nicht bei den bayerischen Wittelsbachern gesucht, für Linderhof aber sehr wohl auf München Bezug genommen werden sollte. Andererseits wurden für die Tmeicos Ettal-Planung noch mehr Mühen aufgewendet als für Linderhof, wie u.a. die Tatsache zeigt, dass der letzte Entwurf sogar in öl gemalt wurde. ${ }^{26}$ Doch kann daraus nicht zwingend gefolgert werden, Linderhof habe bei Bedarf die Funktion einer Probebühne gehabt, wo Ideen für das Hauptprojekt Tmeicos Ettal auf ihre Tauglichkeit geprüft wurden. 


\section{Genetische Wurzeln des Rokoko-Bettes}

16 Der Richtungswechsel beim Bett lässt sich dank einer Notiz in den Kabinettskassenbüchern zumindest rudimentär nachvollziehen. Zahlungsbelege an den Tapezierer Max Steinmetz vermerken: „Zur Anfertigung eines Prachtbettes im Style Louis XV. nach einer flüchtigen Skizze von Director Franz Seitz wurde dieselbe vom Unterzeichneten /:Steinmetz:/ im Auftrage des k. Hofsekretariats nach einem Werke von Meissonier umgearbeitet und nach diesen von Seiner Majestät genehmigten Zeichnungen ausgeführt. ${ }^{{ }^{27}}$ Der Seitz-Entwurf entsprach also nicht den Vorstellungen des Königs vom Rokoko Ludwigs XV. Vielmehr scheint ihm ein Stil wie ihn Juste Aurèle Meissonier verkörperte vorgeschwebt $\mathrm{zu}$ haben. Dessen um 1750 in Paris herausgegebenes Oeuvre mit einer Vielzahl seiner Entwürfe für Goldschmiedearbeiten, Möbel, Raumeinrichtungen und Architektur befand sich im Besitz Ludwigs II. In dem Exemplar, das heute in der Bayerischen Staatsbibliothek verwahrt wird, ${ }^{28}$ finden sich bei 17 Tafeln Notizen, die auf Ludwigs Bau- und Ausstattungsprojekte verweisen. So kann der Gesamtentwurf für das 1872 vollendete Arbeitszimmer aufgrund der Notiz "Arbeitszimmer, grünes" auf Tafel 85, "Projet du Sallon de la Princesse Satorinski en Pologne", zurückgeführt werden, ein "Provisorscher Spiegel für das Schlafzimmer" sollte nach Tafel 87, "Cabinet pour le Comte Bielinski" ausgeführt werden. Keine dieser Notizen bezieht sich auf das Bett, aber die Formensprache seiner Schnitzereien scheint von Meissoniers Stil inspiriert zu sein. Dieser zeigt sich in Motivenwie den fleischigen Rocaillen, kräftigen, gedrungenen C-Bögen oder gestreckten, nur leicht geschweiften Gliedern, die mit einem leichten Rocaillensaum belegt sind, während der Gegenschwung godroniert ist. Auch die Kombination von Rocaillen mit Akanthuswedeln oder der Zopf aus Blattkelchen am Schaft der Puttenhermen der Eckstollen sind bei Meissonier zu finden. ${ }^{29}$ Die auffällig kräftigen Füße, die organisch als Teil der unteren Zarge statt des Eckstollen erscheinen (Abb. 10), erinnern stark an die obere Partie seines Konsoltisch-Entwurfs Tafel 48, "Table d'appartement". ${ }^{30}$ 
Abb. 10

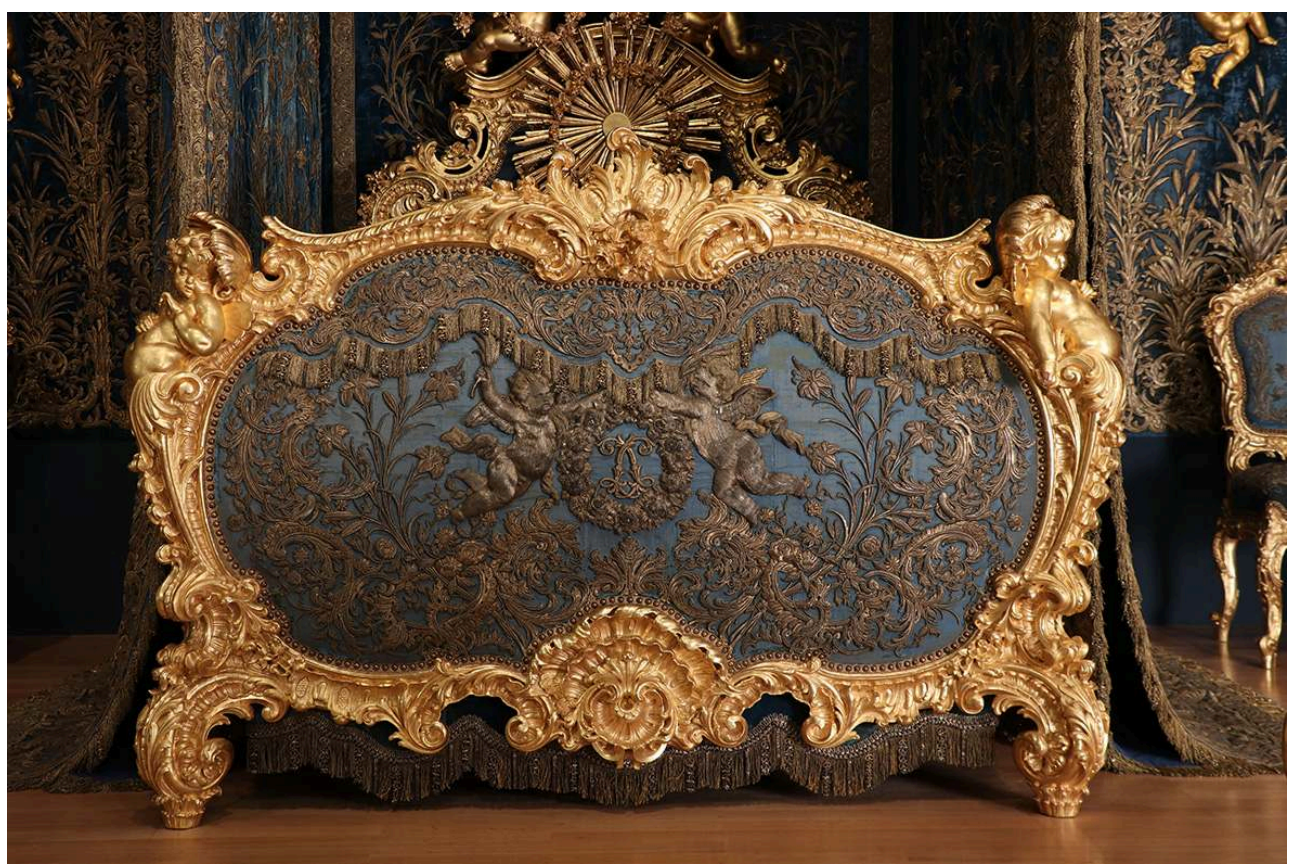

Fußende des Bettes, Zustand 2018

Foto Sybe Wartena. (c) Bayerisches Nationalmuseum

Die Ornamentmotive könnten auch andere Quellen haben, Meissonier ist sicher nur eine Möglichkeit. Wichtig scheint jedoch die Feststellung, dass das Bett im gesamten auf keiner Vorlage aus dem 18. Jahrhundert basieren kann, weder auf einem reellen Möbel noch auf einem Entwurf. Aus dem 18. Jahrhundert sind keine vergleichbar opulent geschnitzten Baldachine oder Bettgestelle bekannt, zumindest nicht in Kombination mit aufwendigen Textilien. ${ }^{31}$ Hohe, reich geschnitzte Aufsätze am Kopfende sind im 17. Jahrhundert verbreitet, aber keine erhöhten Fußenden. Die "verdrehten" Füße oder die Hermen am oberen Ende des geschweiften Eckstollen erinnern an manche Kommoden und Konsoltische des Rokoko, doch für das ganze Fußende scheiden diese als Inspiration aus. Vielmehr erinnert es an Kartuschen, von denen bei Entwerfern des 18. Jahrhunderts und besonders bei Meissonier zahlreiche, sehr ähnliche zu finden sind. ${ }^{32}$ Hier ist zu betonen, dass das Bett der Chambre de Parade von Herrenchiemsee trotz sehr ähnlicher Gliederungsprinzipien in der Formensprache Stilelemente der Jahrzehnte um 1700 aufgreift. Das Bett des kleinen Appartements ist hingegen auch im ornamentalen Detail dem von Linderhof sehr eng verwandt.

\section{Funktionsweise des Bettes und Ikonografie}

Das hohe Fußende lenkt den Blick auf ein funktionales Merkmal, das im 18. Jahrhundert so nicht denkbar wäre. Die Bettseiten sind auffällig niedrig (Abb. 11). ${ }^{33}$ Die Oberkante der Matratze kann nicht erheblich höher gewesen sein, als die oberen Partien der geschweift konturierten Seite. Zwischen der Matratze und der auf einem Lattenrahmen montierten und oben zwischen Kopf- und Fußende aufgehängten Couvertdecke blieb ein beträchtlicher Abstand. Dies ist von Betten des Louis XVI. und des Empire bekannt, ${ }^{34}$ deren Fußende annähernd oder genauso hoch ist wie das Kopfende, nicht aber aus dem Rokoko, wo solche Bettüberwürfe auf der Matratze 
auflagen. Unklar ist in diesem Punkt, wie bei den Entwurfsstadien ohne erhöhtes Fußende dessen Gestaltung gedacht war, oder ob die in den Entwürfen dargestellte textile Sichtseite des Fußendes gar nicht als Behang der Couvertdecke mit abgenommen werden sollte sondern als Teil des Bettgestells gedacht war. Das ausgeführte Linderhofer Bett scheint diesen Gedanken in der Fortführung des oberen Lambrequins von den Seitenbehängen anzudeuten.

Abb. 11

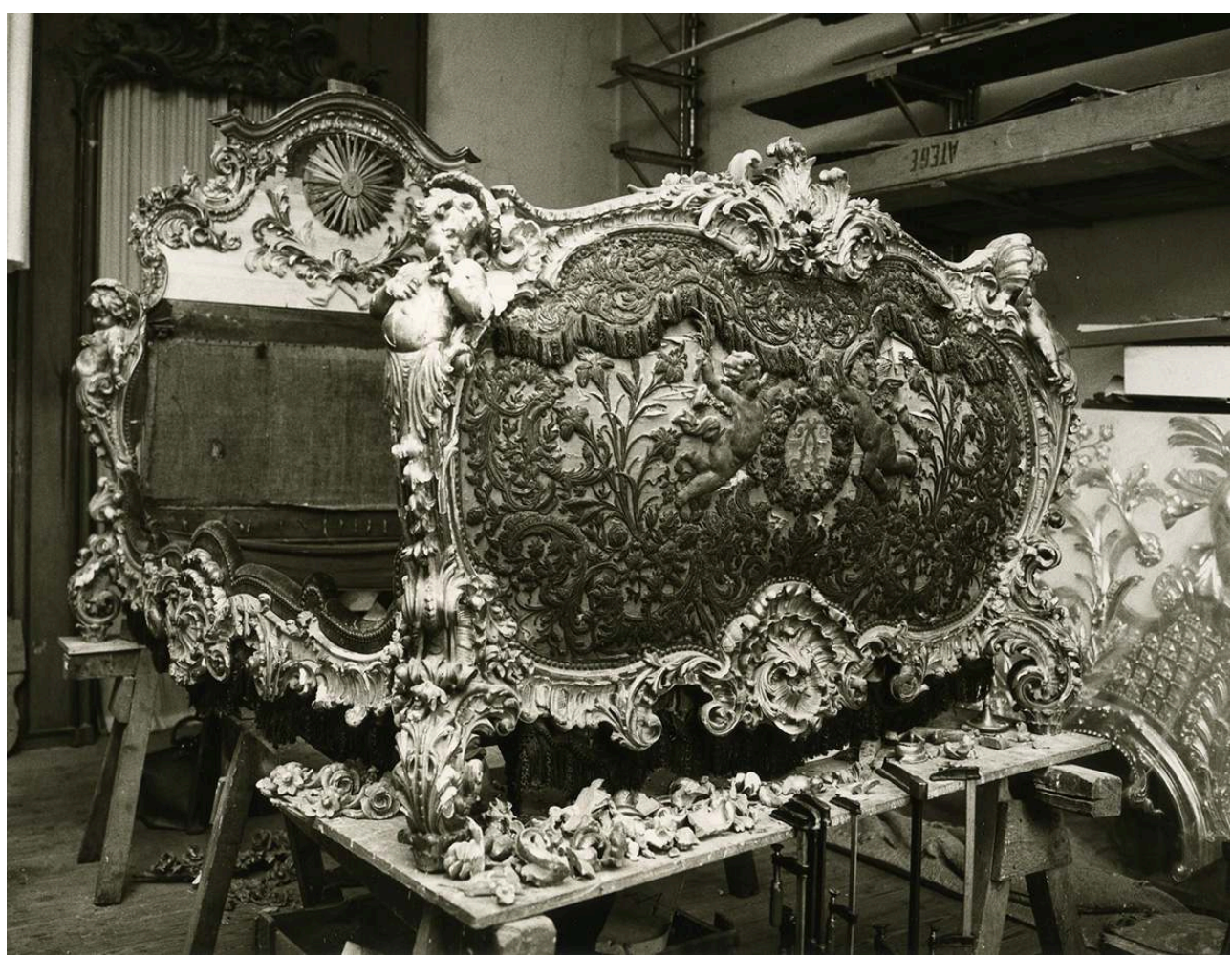

BETTgESTELL, ZUSTAND VOR 1968.

Foto Bayerisches Nationalmuseum. (c) Bayerisches Nationalmuseum.

Die Kombination eines technisch-funktionalen Konzepts des 19. Jahrhunderts mit gestalterischen bzw. ornamentalen Formen früherer Epochen ist typisch für viele der Kunsthandwerklichen Arbeiten für Ludwig II., seien es Kutschen und Schlitten oder Heizungssysteme und Telefonanlagen in seinen Schlössern. So war das Bett, wie auch die beiden Betten auf Herrenchiemsee, mit einer dreiteiligen Matratze aus 60 Pfund Rosshaar und einer "Federmatraze, gepolstert mit Gurten, Federn, Roßhaaren, verschiedenen Leinwänden..." ${ }^{35}$ ausgestattet, womit wohl ein Sprungfederrahmen gemeint ist.

Das Bildprogramm des ersten Linderhofer Bettes ist weit weniger umfangreich als jene der beiden Betten von Herrenchiemsee, doch ist auch hier eine Verwandtschaft festzustellen. Das Bett selbst weist nur die Ludwigs-Initiale, die Bayerische Krone mit Orden, zwischen Ranken flatternde Putti an den Außenseiten der Vorhänge und, im Zentrum des Baldachins, die Waffen Amors auf (Abb. 12). 
Abb. 12

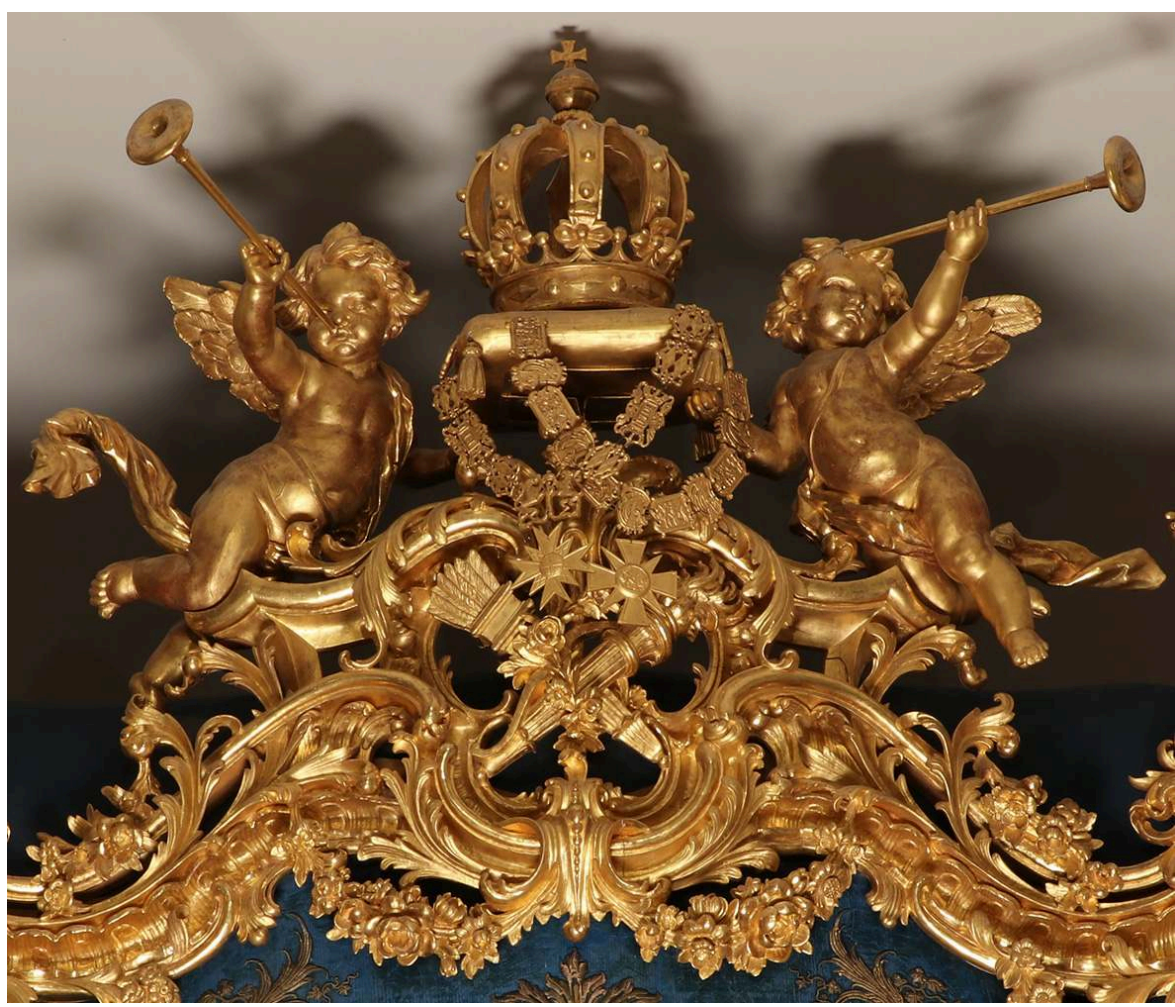

BETTBALDACHIN, MITTELPARTIE DER FRONT, ZUSTAND 2018.

Foto Sybe Wartena. (c) Bayerisches Nationalmuseum.

21 Die angrenzenden, blausamtenen Wandfelder bilden mit ihren in Gold gestickten Rocaillenbordüren, Lilienstauden und Rosengirlanden eine reiche Rahmung für je fünf flatternde Putti aus vergoldeter Bronze. ${ }^{36}$ Als formale Anregung für diese Putti wurden überzeugend die Wanddekorationen des Ruhezimmers in der Amalienburg im Nymphenburger Schlosspark angenommen, ${ }^{37}$ doch anders als dort, sind hier nicht die vier Elemente gemeint. Ihr Ausdruck ist als ausgelassen, neckisch und keck zu bezeichnen. Einige musizieren auf Zimbeln, winzigen Geigen und Trompeten, andere lassen einen Drachen steigen, spielen mit einem Papagei oder tanzen. Sie fügen sich bestens zu den Figurengruppen auf dem Deckengesims: dort waren 1871 vom Bildhauer Franz Walker fünf mythologische Liebespaare als vergoldete Stuckreliefs geschaffen worden: Amor und Psyche, Bacchus und Ariadne, Venus und Mars, „Diana betrachtet den schlafenden Endymion“ und „Venus liebkost Adonis“. ${ }^{38}$ Wie eine nachträgliche Bestätigung scheint die Anordnung Ludwigs vom 12. August 1872, dass „alles, was auf Liebe Bezug hat (im mythologischen Sinne) ins Schlafzimmer kommen solle". ${ }^{39}$ Das Bett der Chambre de Parade in Herrenchiemsee wies nicht weniger als 23 Szenen zum Thema der Macht der Venus auf, das Bett des Schlafzimmers des kleinen Appartements weist am Fußende Venus und Adonis an den Pfosten auf, dazwischen die ruhende Venus in Relief, an der Rückwand jedoch den Triumph Ludwigs XIV. über das Laster.

Die gestickte Ornamentik der Wandfelder, welche das Reinheitssymbol der Lilie als Hauptmotiv verwendet, mag als Kontrast zu der von den Liebesgruppen und Putti oder Eroten evozierten Stimmung gedacht gewesen sein. Noch deutlicher scheint die Darstellung am Betthimmel in diese Richtung zu deuten: Hier ist die Taube des Heiligen Geists mit einer Ampulle im Schnabel dargestellt (Abb. 13). 
Abb. 13

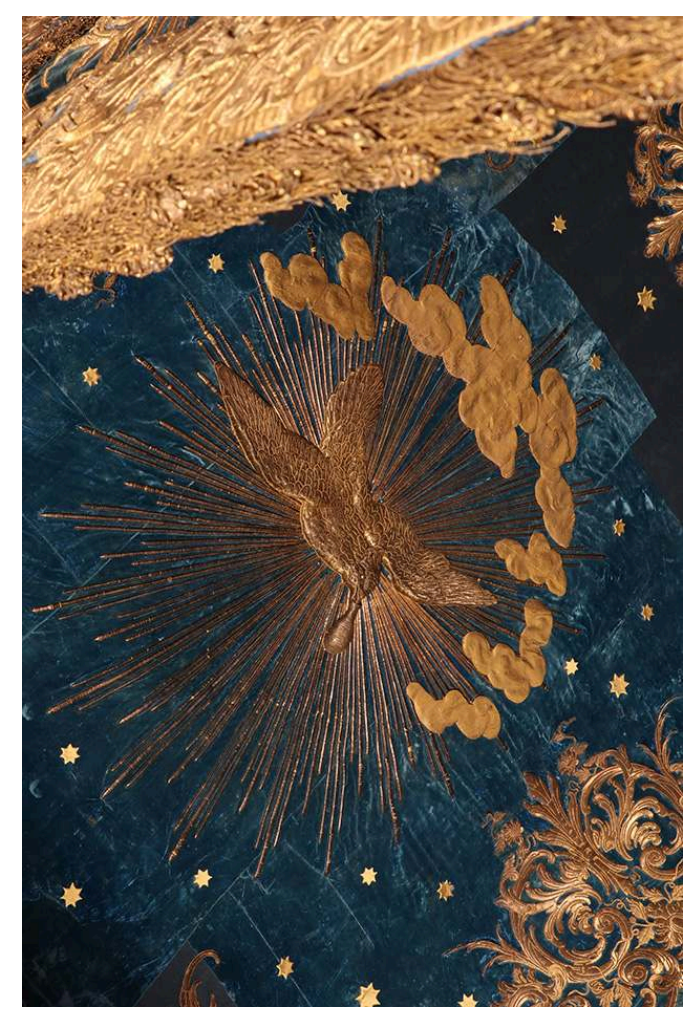

BETTBALDACHIN, UNTERSEITE: HEILIg-GEIST-TAUBE MIT SALBÖL-AMPULLE (OBEN IM BILD: RECHTER BETTVORHANg), ZUSTAND 2018.

Foto Sybe Wartena. (c) Bayerisches Nationalmuseum.

Dieses Motiv kehrt 1884 in Herrenchiemsee mit dem Bett des Schlafzimmers im kleinen Appartement wieder. ${ }^{40}$ Ein weiteres Mal tritt es zwischen 1875 und 1881 an einer Wechseldarstellung für die Rückwand des Bettes der großen Chambre de Parade in Herrenchiemsee auf. Dieses Bild, „Le vœu de Louis XIV.“ in der Technik der sogenannten Nadelmalerei, konnte eingesetzt werden, um die Flügelbeschneidung Amors durch Nymphen zu verdecken. ${ }^{41}$ Die Darstellung zeigt Ludwig XIV., der seine Krone der Muttergottes weiht, während der Jesusknabe von der heranfliegenden Heilig-Geist-Taube die Ampulle mit dem Salböl empfängt. Dass die Hoffnung auf göttlichen Beistand für sein Ringen um ein ideales Königtum für Ludwig II. ein wichtiges Thema war, wurde u.a. aus seinen Tagebucheinträgen deutlich. Schon in seinem Schlafzimmer in der Münchner Residenz hing neben seinem Bett ein Gemälde der Salbung und Krönung Ludwigs XIV., auf Französisch „sacre“, welches Ludwig II. wohl auch als Heiligung des gottgewollten Königtums verstand. Das Thema der Salbung ist somit eine Konstante in den „französischen“ Schlafzimmern König Ludwigs, und die Taube mit dem Salböl tritt zuerst am Linderhofer Bett auf.

\section{Die Aufgabe des ersten Linderhofer Schlafzimmers und seine museale Geschichte}

1884 ordnete König Ludwig II. an, das Linderhofer Schlafzimmer zu vergrößern. ${ }^{42}$ Schon im ersten Nachweis für die Planung wird klar, dass eine enge Anlehnung an das 
Schlafzimmer der Reichen Zimmer des nachmaligen Kaisers Karl Albrecht in der Münchner Residenz angestrebt wurde, denn die Maße des dortigen Bettes wurden für dieses Projekt aufgenommen und übermittelt, ebenso wie die Gesamtmaße des Schlafzimmers der Reichen Zimmer und des ersten Linderhofer Schlafzimmers im Vergleich ${ }^{43}$ sowie die beider Betten bzw. Baldachine. ${ }^{44}$ Ferner hatte Ludwig II. angeordnet, dass die Disposition von Fenstern und Türen vom Schlafzimmer der Reichen Zimmer übernommen werden musste und "daß die Stickerei der Wände genauso wie im hiesigen Reichen Schlafzimmer $\mathrm{zu}$ werden hat". ${ }^{45}$ Eine engere Übereinstimmung liegt nur bei den geschnitzten Wandvertäfelungen und dem Deckenstuck vor, aber eine grundsätzliche Orientierung ist auch bei den Wandbespannungen zu erkennen. Tatsächlich nähern sich die Maße des zweiten Bettes mit einer Grundfläche von 255 x $300 \mathrm{~cm}^{46}$ denen des Bettes der Reichen Zimmer an, die nach der Messung von 1884 bei ca. 238 x $311 \mathrm{~cm}$ liegen; das erste Bett misst hingegen nur 180 x $243 \mathrm{~cm}$ in der Fläche. Als die Arbeiten schon in vollem Gange sind, folgt noch die Anordnung "Der Plafond des Betthimmels, ebenfalls die Taube mit dem Oehlfläschchen, Strahlen, Sterne und Wolken", ${ }^{47}$ womit die Bedeutung dieser Ikonographie nochmals unterstrichen wird.

Ein eigenständiger Entwurf für das Bett ist nicht erhalten, ${ }^{48}$ wenngleich überliefert ist, dass dem König Entwürfe zur Bewilligung vorgelegt wurden. ${ }^{49}$ Das einzige bekannte relevante Blatt von Eugen Drollinger $1884^{50}$ (Abb. 14) entspricht außer in den Proportionen in nahezu allen Details dem ausgeführten ersten Bett.

Abb. 14

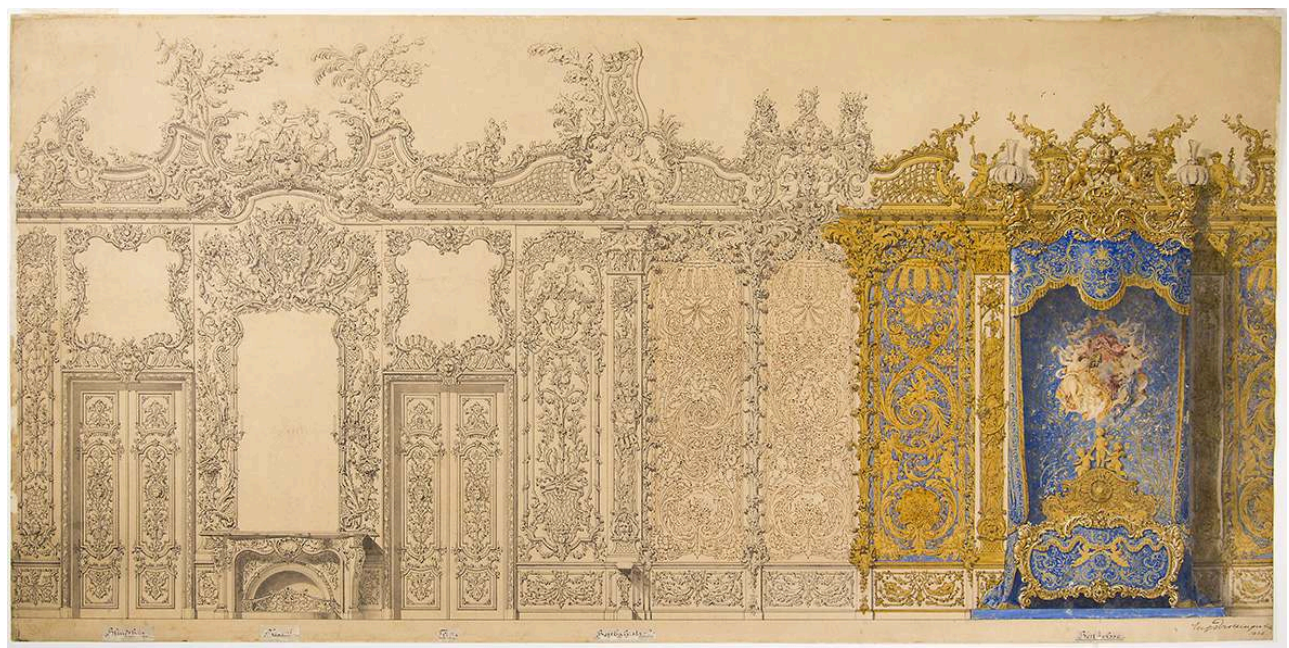

ENTWURF FÜR DAS ZWEITE LINDERHOFER SCHLAFZIMMER, WANDABWICKLUNG. EUgEN DROLLINGER, 1884. FEDER, TEILWEISE AQUARELLIERT, 45,3 x 92 CM. BSV, L.II.-MUS. 268.

(C) Bayerische Schlösserverwaltung. www.schloesser.bayern.de.

Die einzige nennenswerte Abweichung ist die stärker bewegte obere Konturlinie des Fußendes, die beiderseits der zentralen Akanthusmuschel aus zwei kräftigen gedrungenen C-Bögen statt eines leichteren, langgezogenen gebildet werden sollte. Und gerade hier gab es bei der Ausführung des zweiten Bettes die einzige größere Abweichung vom Entwurf Drollingers, die durch die "Allerhöchsten Befehle die Einrichtungsgegenstände des Schlafzimmers Linderhof betreffend" erhellt werden, welche Architekt Julius Hofmann am 27. März 1886 Kammerlakai Lorenz Mayr übermittelte: "... Die Decke des Bettes müsse vorne und an den Seiten herabhängen." ${ }^{1}$ 
Wohl um dies zu ermöglichen - keine andere Erklärung erscheint plausibel - wurde der obere Abschluss des Fußendes radikal verändert: statt der kräftigen C-Bögen mit reichen Akanthusblättern bzw. Rocaillen, die hoch über die Köpfe der seitlichen Putten hinausragen sollten, wurde das Fußende in Höhe der Puttenköpfe glatt abgeschnitten und der Rahmenfries mit kleinteiliger Ornamentik gefüllt, die nach unten eine unruhige, unorganische Linie ergibt (Abb. 15). Diese unglückliche Lösung, die neben dem oberen Abschluss auch die Proportionen des so wichtigen Fußendes betrifft, wäre zwar kaschiert worden, wenn die Couvertdecke nicht nur mit Behängen an den Seiten (Ausführung) sondern wie angeordnet auch am Fußende realisiert worden wäre - doch stehen die Schnitzereien weit vor die waagerechte Abschlussleiste vor, sodass zumindest eine weitere Hilfskonstruktion nötig gewesen wäre, um ein freies Herabhängen zu ermöglichen. Die unbefriedigende Wirkung wird dadurch verstärkt, dass die Füllung aus Gründen der Ersparnis mit schlichtem blauem Samt geschlossen und auf die geplanten Goldstickereien verzichtet wurde. Zusammen mit der Unsicherheit, die nach Ausweis der Entwurfsstadien von 1871/72 hinsichtlich der Höhe des Fußendes und der Frage, ob die Couvertdecke auch am Fußende einen Behang haben sollte oder nicht, drängt sich der Eindruck auf, dass die Konsequenzen dieses Punktes in der Kommunikation zwischen dem König und den ausführenden Künstlern und Kunsthandwerkern unberücksichtigt blieben. ${ }^{52}$

Abb. 15

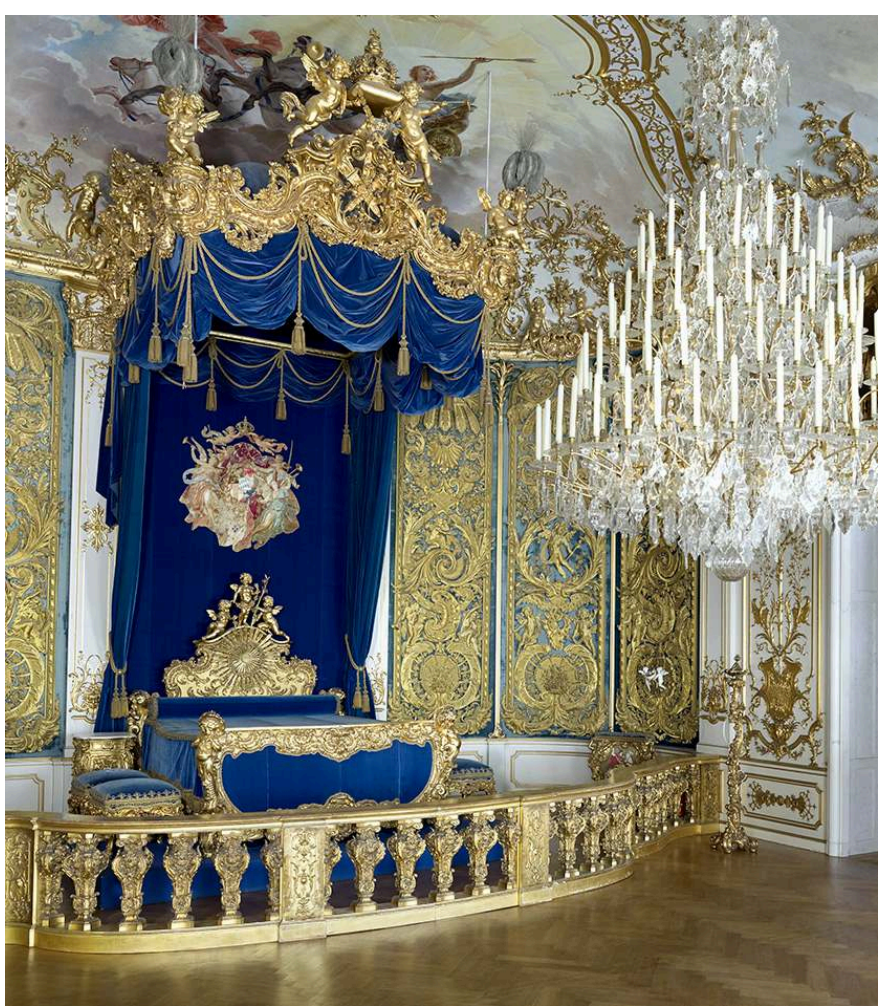

ZWEITES LINDERHOFER SCHLAFZIMMER, ZUSTAND 2018.

(C) Bayerische Schlösserverwaltung. www.schloesser.bayern.de.

Am 24. Oktober 1887, also gut 16 Monate nach dem Tod König Ludwigs II., wurden das Bett, sämtliche Textilien, der geschnitzte Baldachin in 18 Einzelteilen, die Wandbespannungen, vier zum Schlafzimmer gehörende Stühle und zwei Tabourets 
sowie die Fenstervorhänge und Portièren vom königlichen Hofsekretariat an das Bayerische Nationalmuseum abgegeben, welches das Ensemble umgehend in Saal $23 \mathrm{im}$ I. Stock des Altbaus des Museums in der Maximilianstraße ausstellte. ${ }^{53}$ Ein fehlender Abschnitt der Baldachinumrahmung konnte durch einen Hinweis des Hofrats von Klug von der königlichen Vermögensverwaltung im Straßburger Kunstgewerbemuseum, welches etliche Gegenstände aus dem Nachlass Ludwigs II. erworben hatte, ausfindig gemacht und Ende 1887 zurückgeholt werden. Schon 1888 wurde das Bett auf der Deutsch-Nationalen Kunstgewerbeausstellung in München in einem eigenen Pavillon zu Ludwig II. präsentiert ${ }^{54}$ und 1893 wurde es im Saal des Bayerischen Kunstgewerbevereins der Weltausstellung in Chicago ausgestellt. ${ }^{55}$ Am 29. Juli 1894 folgte eine weitere Übereignung: "Sechs geschnitzte und vergoldete Palmen aus dem alten Schlafzimmer des Schlosses Linderhof aus der Vermögensadministration des Königs Otto". .56

Im 1900 eröffneten Neubau des Museums an der Prinzregentenstraße wurde Saal 48 König Ludwig II. gewidmet. Neben den Bestandteilen des Schlafzimmers wurden das bekannte Staatsporträt des zwanzigjährigen Ludwig II. von Ferdinand von Piloty, eine Büste Richard Wagners und wenige weitere Objekte ausgestellt. Interessant ist die architektonische Einbindung, die den Salon de l'Oeil de Boeuf zitierte und beiderseits des Bettes große Spiegelachsen einführte, aber auf charakteristische Merkmale wie die Rundung der Bettnische oder die geschwungene Balustrade verzichtete (Abb. 16). Stattdessen wurden monumentale korinthische Säulen aus Stuckmarmor verwendet, die an das opulenteste Planungsstadium des Schlafzimmers für Herrenchiemsee mit nicht ausgeführten Säulen als Zäsur am Eingang der Bettnische erinnern.

Abb. 16

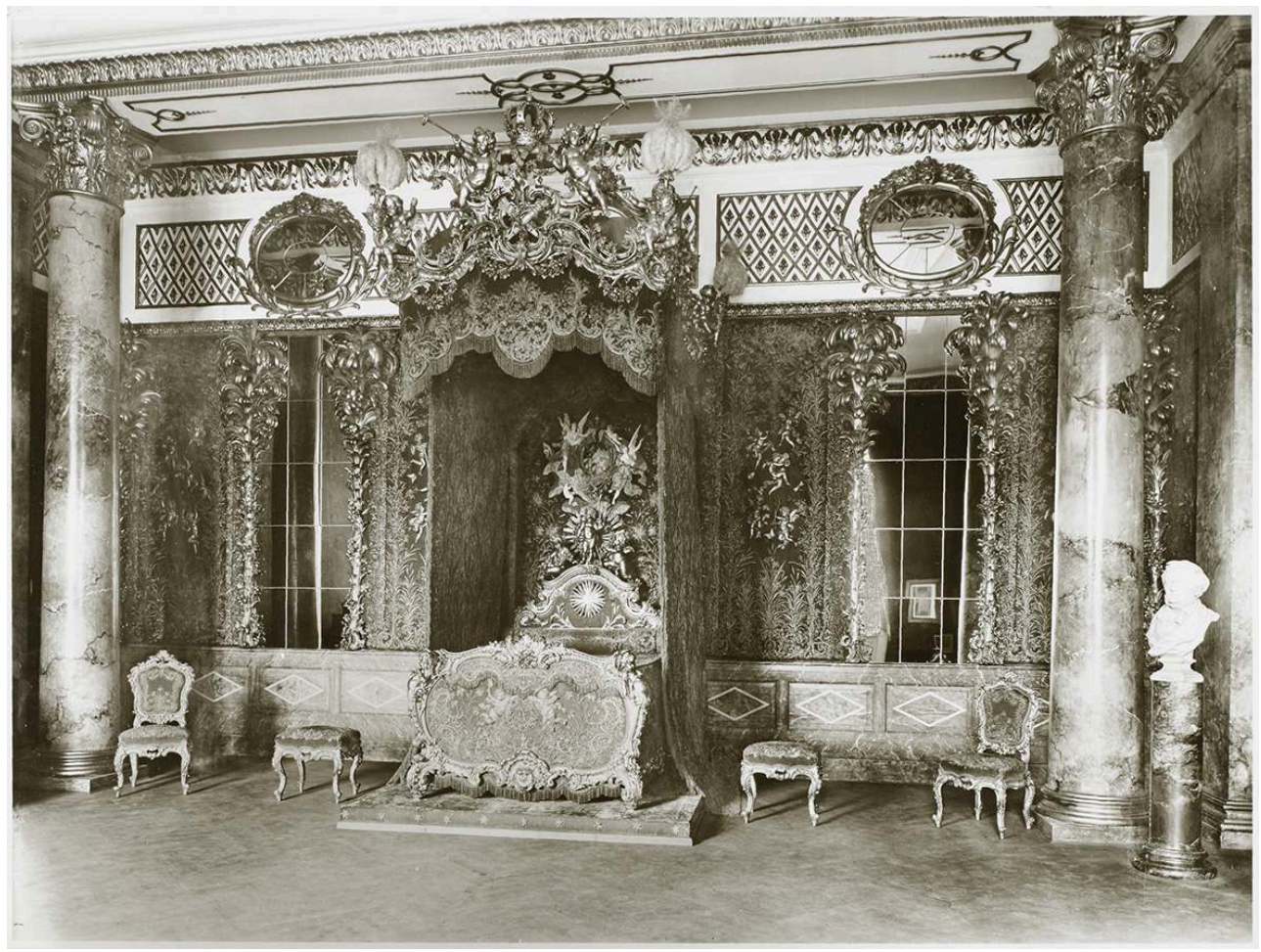

BAYERISCHES NATIONALMUSEUM, SAAL LUDWIgS II. (SAAL 48), ZUSTAND 1908 ODER 1909.

Foto Bayerisches Nationalmuseum. (c) Bayerisches Nationalmuseum. 
1908 gelang dem Museum eine wichtige Erwerbung zur Vervollständigung des Schlafzimmers: die 25 Putti aus feuervergoldeter Bronze einschließlich sieben zugehöriger Schmetterlinge und fünf Wasserjungfern (Libellen), die ebenfalls vom Straßburger Kunstgewerbemuseum angekauft worden waren. ${ }^{57} \mathrm{Nach}$ dem zweiten Weltkrieg wurde die chronologische Gliederung des Rundgangs mit Sälen zu den jeweiligen Herrscherpersönlichkeiten aufgegeben (sie wurde in Teilbereichen 2015 wiederhergestellt). Erst zur Ausstellung "König Ludwig II. und die Kunst" in der Münchner Residenz 1968 trat das erste Linderhofer Schlafzimmer wieder ins Bewusstsein. Zu dieser Gelegenheit kam das Bett, das erhebliche Fassungsschäden und die Ablösung etlicher Teile der Schnitzerei erlitten hatte, als Leihgabe an die Bayerische Schlösserverwaltung und wurde restauriert. Seit der Neueinrichtung des König Ludwig II.-Museums in Herrenchiemsee 1986 ist es dort in einer dem Originalzustand in dem wichtigen Punkt der Rundung der Bettnische angeglichenen Zustand ausgestellt. Dadurch werden die Raumproportionen korrekt angedeutet, während durch das Fehlen von Gesims und Decke die nötige Abstraktion erreicht wird, um den fragmentarischen Charakter zu vermitteln (Abb. 17, Abb. 18).

Abb. 17

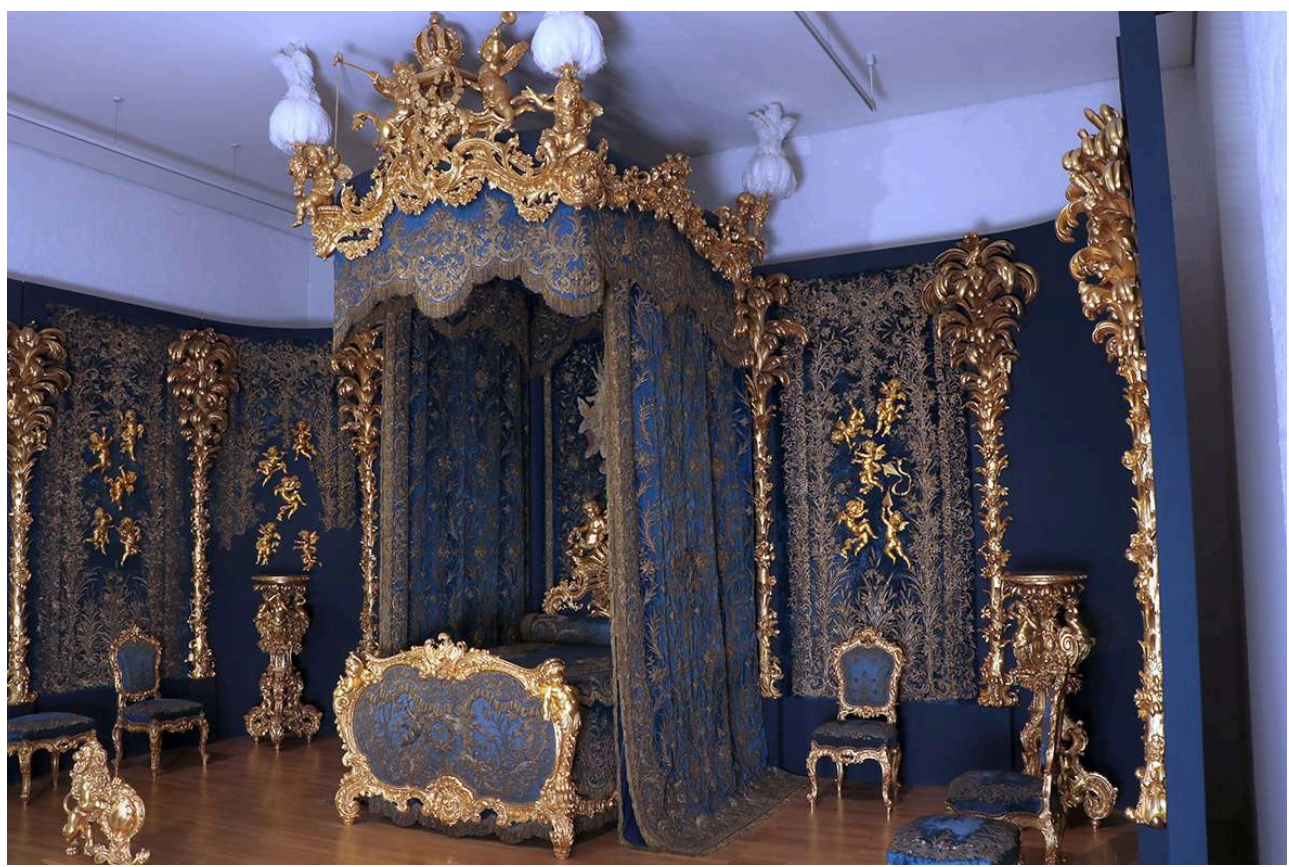

PRÄSENTATION DER ERHALTENEN SCHLAFZIMMERAUSSTATTUNg IM LUDWIg. II.-MUSEUM, GESAMTANSICHT. ZUSTAND 2018.

Foto Sybe Wartena. (c) Bayerisches Nationalmuseum. 
Abb. 18

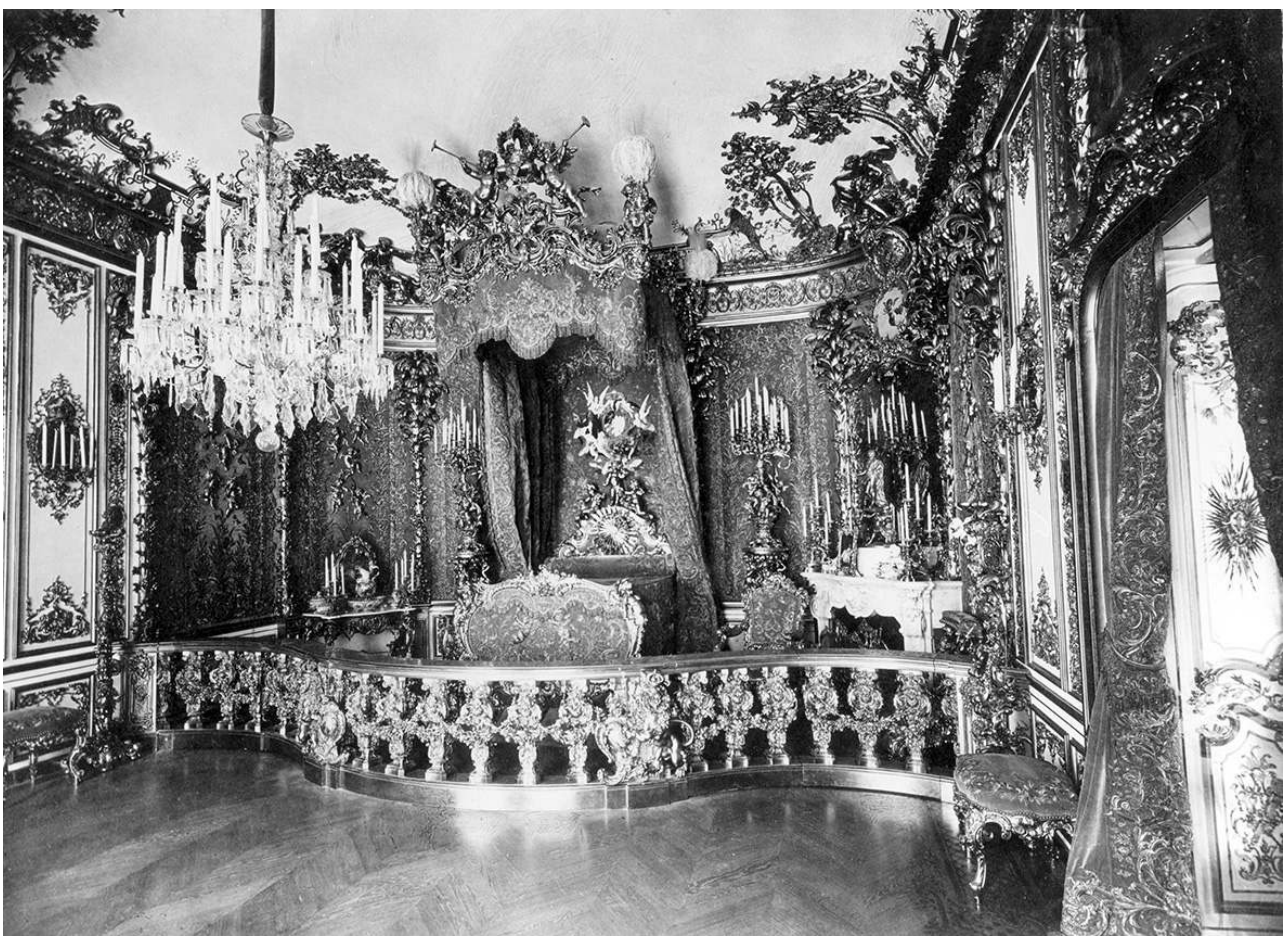

ERSTES LINDERHOFER SCHLAFZIMMER, PHOTOgRAPHIE JOSEPH ALBERT, VOR 1878.

(C) Bayerische Schlösserverwaltung. www.schloesser.bayern.de.

\section{NOTES}

1. - Bayerisches Nationalmuseum Inv.-Nr. T 2468; dazu zahlreiche Zubehörteile, Wandbespannung, zugehörige Sitzmöbel mit eigenen Inventarnummern.

2. - Baumgartner, Georg. Königliche Träume. Ludwig II. und seine Bauten. München 1981. Heinrich Hugendubel Verlag. S. 186; Petzet, Michael. Gebaute Träume. Die Schlösser Ludwigs II. von Bayern. München 1995. Hirmer Verlag. S. 136.

3. - Im Widerspruch zu dieser Planungsphase scheint die These zu stehen, Linderhof sei im Verhältnis zu Tmeicos Ettal nach Versailler Vorbild die Rolle eines Trianon als Rückzugsort zugedacht gewesen (Bachmayer, Monika. Schloss Linderhof. Architektur, Interieur und Ambiente einer „Königlichen Villa“. München 1977 (Phil. Diss. München, S. 59-60). Vielmehr erscheint sie wie ein Notplan für den Fall des Scheiterns des Hauptprojekts. Dennoch liegen bei den Schlafzimmern beider Projekte Zusammenhänge vor.

4. - Zitate nach der Münchner Residenz modifizieren nur bedingt die Feststellung, dass Linderhof (wie Tmeicos Ettal/Herrenchiemsee) Kulisse für Idealbilder des französischen Absolutismus werden sollte, denn die gesamte Ausstattung Linderhofs ist diesem Feld gewidmet.

5. - Sailer, Ludwig. Die Bau- und Kunst-Denkmäler weiland Sr. Majestät König Ludwig II. von Bayern: ein Begleiter und andenken für den Besucher der königl. Schlösser Chiemsee, Linderhof, Neuschwanstein, Hohenschwangau und Berg. München 1886. Verlag Frisch. S. 46. 
6. - Baumgartner 1981, S. 49.

7. - Baumgartner 1981, S. 43 Abbildung 40; Bestandteile des Bettes ausgestellt im Ludwig II.Museum, Schloss Herrenchiemsee: Hoyer, Gerhard (Hrsg.). König Ludwig II.-Museum Herrenchiemsee. Katalog. München 1986. Hirmer Verlag. S. 165.

8. - Wartena, Sybe. Souvenirs historiques des Résidences Royales de France, Bd. 1: Palais de Versailles./"Chambre à coucher de Louis XIV"/Die Chambre de Parade in Versailles. In: AK Götterdämmerung 2011. S. 120-121, 139-140 (Kat.-Nr. 3.25a, 3.25c, 3.38a).

9. - „Versailles - Herrenwörth. Bettbeschreibung“ Wittelsbacher Ausgleichsfonds, Manuskript 45. 10. - Wartena, Sybe. Entwurf für das Paradebett des Prunkschlafzimmers - Vorderansicht. In: AK Götterdämmerung 2011. S. 139-140 (Kat. 3.38b).

11. - Abbildungen: Seitenansicht: Baumgartner 1981, S. 136, Abb. 237; Vorderansicht: AK Götterdämmerung 2011. S. 139 (Kat. 3.38b). Erster Entwurf mit vier Stützen unveröffentlicht.

12. - $\mathrm{Zu}$ den Textilien ist eine große Zahl aufwendigster Entwürfe erhalten geblieben, die hier nicht thematisiert werden sollen.

13. - Baumgartner 1981, S. 153, Abb. 266, Petzet 1995, S. 235.

14. - Bayerisches Hauptstaatsarchiv (BHStA), Geheimes Hausarchiv (GHA), Hofsekretariat 385. Haupt-Rechnung des Cabinets-Casse Seiner Majestät des Königs Ludwig II. für das Jahr (künftig: Hauptrechnung) 1871. Nebenrechnung der koenigl. Cabinetts-Cassa über die Ausgaben für das kgl. Besitzthum „Linderhof“" (künftig Nebenrechnung Linderhof) pro 1871, S. 26, Beleg Nr. 102.

15. - In den Rechnungen ist immer von „Bettdeckel“ die Rede; hier soll nach Langer Brigitte, Die Möbel der Schlösser Nymphenburg und Schleißheim. München 2000. Prestel Verlag. S. 98, eine der Bezeichnungen des 18. Jahrhunderts verwendet werden: Couvertdecke.

16. - De Soucy. Ameublement du Roi, pour son Grand Appartement de Versailles, fait par Simon Delobel, tapissier et valet de chambre de sa Majesté et garde des meubles de la reine. In. Vatout. Jean, Le Palais de Versailles, S. 419-429, 422, 425.

17. - Der Entwurf zeigt zwar nicht die Dopplung der Vorhänge, doch führte wohl die technische Ausführung zwangsläufig zu zwei getrennten Lagen. Vgl. die Goldvorhänge des Bettes der Chambre de Parade auf Herrenchiemsee. Heinemann, Katharina und Wartena, Sybe. Goldvorhang des Prunkbettes im Paradeschlafzimmer in Schloss Herrenchiemsee. In: AK Götterdämmerung 2011, Kat.-Nr. 3.43, S. 145-146.

18. - BHStA, GHA, Hofsekretariat 386. Hauptrechnung 1872. Nebenrechnung Linderhof 1872, S. 28, Beleg Nr. 168.

19. - BHStA, GHA, Hofsekretariat 385. Hauptrechnung 1871. Nebenrechnung Linderhof 1871, S. 26, Beleg Nr. 98.

20. - Schmid, Elmar D. Schloß Linderhof, erstes Schlafzimmer. Entwurf zum Prunkbett. In: Hoyer, Gerhard (Hrsg.). König Ludwig II.-Museum Herrenchiemsee. Katalog. München 1986. Hirmer Verlag. S. 354, Kat.-Nr. 248.

21. - Schick, Afra. Möbel für den Märchenkönig. Ludwig II. und die Münchner Hofschreinerei Anton Pössenbacher. Stuttgart 2003. Arnoldsche Verlagsanstalt. S. 210-211; Saalbuch Bayerisches Nationalmuseum Saal 19-23, ab 1881, S. 95.

22. - Die 1872 für Tmeicos Ettal dokumentierte Form könnte bereits früher in nicht erhaltenen Entwürfen entstanden sein.

23. - Bachmayer, Monika. Schloss Linderhof. Architektur, Interieur und Ambiente einer „Königlichen Villa“. München 1977 (Phil. Diss. München). S. 235, Quellenbeleg Nr. 5.6.1.2, Bayerische Schlösserverwaltung Inv.-Nr. L.II.-Mus Nr. 2000.

24. - BHStA, GHA, Hofsekretariat 386. Hauptrechnung 1872. Nebenrechnung Linderhof 1872, S. 29, Beleg Nr. 169.

25. - Goertz, Beate. Schloss Linderhof. Die Innenraumgestaltung der beiden Schlafzimmer. [MS] Magisterarbeit Universität Wien 1996, S. 67, Anm. 226. 
26. - Heinemann, Katharina. Entwurf für die Chambre de Parade in Herrenchiemsee. In: AK Götterdämmerung 2011. S. 140-141. Das Ölgemälde von Wilhelm Hauschild kann wahrscheinlich der Zahlung von $1200 \mathrm{fl}$. „für die Herstellung eines Ölgemäldes, „das Innere eines Schlafzimmers darstellend“ zugeordnet werden, die 1873 für Linderhof verbucht wurde (BHStA, GHA, Hofsekretariat 387. Hauptrechnung 1873. Nebenrechnung Linderhof 1873, S. 71, Beleg Nr. 158).

27. - Schick 2003, S. 210 (Nach: BHStA, GHA, Hofsekretariat 387. Hauptrechnung 1873. Nebenrechnung Linderhof 1873. Beleg 244).

28. - Bayerische Staatsbibliothek, Rar. 2160; http://daten.digitale-sammlungen.de/ db/0009/ bsb00092646/images/

29. - http://daten.digitale-sammlungen.de/0009/bsb00092646/images/index.html? id $=00092646 \&$ seite $=65 \&$ fip $=193.174 .98 .30 \&$ nativeno $=\% 2 F \&$ groesser $=200 \% 25$.

30. - http://daten.digitale-sammlungen.de/0009/bsb00092646/images/index.html? id=00092646\&groesser $=\&$ fip=193.174.98.30\&no=\&seite=39.

31. - Hojer, Gerhard. König Ludwig II. - ein Bauherr des Historismus. In: Ders. (Hg.). König Ludwig II.-Museum Herrenchiemsee. Katalog. München 1986. Hirmer-Verlag. S. 11-30. S. 27 (Abb. 14), S. 28. Der von Hojer abgebildete Entwurf für ein Prunkbett auf der Londoner Weltausstellung von 1851 nimmt Merkmale des Linderhofer Bettes vorweg und wird sicher zurecht als eine mögliche konkrete Inspirationsquelle angesehen, zumal die einschlägigen Publikationen den für Ludwig II. tätigen Künstlern bekannt waren.

32. - http://daten.digitale-sammlungen.de/0009/bsb00092646/images/index.html? id=00092646\&seite=15\&fip=193.174.98.30\&nativeno=\%2F\&groesser=100\%25; http://daten.digitalesammlungen.de/0009/bsb00092646/images/index.html?

$\mathrm{id}=00092646 \&$ seite $=25 \&$ fip $=193.174 .98 .30 \&$ nativeno=\%2F\&groesser $=100 \% 25$.

33. - Fotografie des Bettgestells vor dem Vergolden: https://radspieler.com/firmengeschichte/ Die Tatsache, dass die Seite mit einem Tuch verschlossen ist, lässt vermuten, dass der Blick in das offene Bett tabu war.

34. - In den Münchner Schlössern standen zu Zeiten Ludwigs II. und zum Teil noch heute einige solcher Betten aus der Werkstatt Georges Jacob, Paris, um 1780 (Langer, Brigitte. Die Möbel der Residenz München I. Die französischen Möbel des 18. Jahrhunderts. München 1995. Prestel: Kat.Nr. 47, 86, 87), und der Münchner Hofschreinerei Daniel um 1815 (Langer, Brigitte u.a.. Die Möbel der Residenz München III. Möbel des Empire, Biedermeier und Spätklassizismus. München 1997. Prestel-Verlag. Kat.-Nr. 31; Langer, Brigitte. Die Möbel der Schlösser Nymphenburg und Schleißheim. München 2000. Prestel-Verlag. Kat.-Nr. 101).

35. - BHStA, GHA, Hofsekretariat 387. Hauptrechnung 1873. Nebenrechnung Linderhof 1873. Beleg 244 (Auslassung in der Widergabe dieses Belegs bei Schick 2003, S. 210).

36. - Arbeiten des Goldschmieds Ferdinand Harrach nach Modellen von Philipp Perron (Selig, Lorenz. König Ludwig II. von Bayern. In: Weltkunst 1986 Heft 24, S. 3895-3897).

37. - Goertz, 1996, 50, Anm. 166.

38. - BHStA, GHA, Hofsekretariat 385. Hauptrechnung 1871. Nebenrechnung Linderhof 1871.S. 22, Belege 82 und 83, Zahlungen von 5 Mal fl. 250 an Bildhauer F. Walker.

39. - Ewers, Hans Gerhard. Ludwig II. von Bayern. Theaterfürst - König - Bauherr. Gedanken zum Selbstverständnis. München 1986, Hirmer-Verlag. S. 217. (nach: BHStA, GHA, Kabinettsakten Ludwigs II., K 55, L 4, Nr. 51).

40. - Schick 2003, S. 232; Entwurf im Ludwig II.-Archiv der Bayerischen Schlösserverwaltung; Heinemann, Katharina. Schlafzimmer Ludwigs II. in Herrenchiemsee: Vorentwurf zum Bett Ludwigs II. In: AK Götterdämmerung 2011. S. 150.

41. - Erichsen, Johannes. Ludwig XIV. weiht der Muttergottes seine Krone („Le vœu de Louis XIV.“). In: AK Götterdämmerung 2011. S. 146-147.

42. - Greifbar ist der Auftrag erstmals über den Bericht des 4. Oktober von Hofsekretär Hornig an den König, worin er die Unmöglichkeit beschreibt, für die Vergrößerung des Schlafzimmers 
lediglich eines der flankierenden "weißen Kabinette" zu opfern (BHStA GHA, Kabinettsakten König Ludwigs II., Nr. 273. Nach Goertz 1996 S. 125-126, Quelle Nr. 7).

43. - BHStA GHA, Kabinettsakten König Ludwigs II., Nr. 273. Nach Goertz 1996 S. 126, Quelle Nr. 8.

44. - BHStA GHA, Kabinettsakten König Ludwigs II., Nr. 273. Nach Goertz 1996 S. 126-127, Quelle Nr. 10.

45. - BHStA GHA, Kabinettsakten König Ludwigs II., Nr. 273. Nach Goertz 1996 S. 126, Quelle Nr. 8.

46. - Inventar BSV.

47. - BHStA GHA, Kabinettsakten König Ludwigs II., Nr. 286. Nach Goertz 1996 S. 133, Quelle Nr. 32.

48. - Ausnahme: nicht ausgeführter Entwurf für den Lambrequin des Betthimmels nach von Eugen Drollinger, nach Schick 2003 S. 212.

49. - BHStA GHA, Kabinettsakten König Ludwigs II., Nr. 278. Nach Goertz 1996 S. 128, Quelle Nr. 15: Hofsekretär Hornig an König Ludwig II. am 15. Februar 1885.

50. - BSV, L.II.-Mus. Nr. 268.

51. - BHStA GHA, Kabinettsakten König Ludwigs II., Nr. 286. Nach Goertz 1996 S. 133, Quelle Nr. 32.

52. - Die Entwürfe für beide Betten in Herenchiemsee weisen in demselben Punkt Unsicherheiten auf.

53. - Bayerisches Nationalmuseum Zugangsbuch I 1883-88, S. 370-371.

54. - Salvisberg, Paul von und andere ( $\mathrm{Hg})$. Officieller Katalog der Deutsch-Nationalen Kunstgewerbeausstellung zu München 1888. München 1888, Commissions-Verlag der Academischen Monatshefte. S. 57, abgebildet als Fig. 81. Getrennt vom Bett waren die Wandpanneaux des neuen Schlafzimmers ausgestellt, kombiniert mit den geschnitzten Palmen des alten; ob die Wandbespannung im Bereich des Bettes identisch ist mit der zugehörigen des ersten Schlafzimmers, ist auf dem Foto nicht eindeutig zu erkennen.

55. - Korrespondenz mit dem Bayerischen Kunstgewerbeverein, Notiz vom 18. Januar 1983 in den Erwerbungsakten des BNM vor 1945, Dok. 1743, König Ludwig II.-Ensemble (Bett). Die Berichterstattung in der Zeitschrift des bayerischen Kunst-Gewerbe-Vereins in München 1893, Beiblatt Nr. 2, S. 13, erwähnt für den von Gabriel von Seidl errichteten Pavillon zwar nur: „Die ... goldgestickten Wandbehänge aus blauem Sammt (ursprünglich in Schloss Linderhof) wurden durch Vermittlung des kgl. Ministeriums vom Nationalmuseum hergeliehen; das Mobiliar und die sonstige Zimmerausstattung aus dem Schloss Herrenchiemsee wurde von der kgl. Vermögensverwaltung zur Verfügung gestellt.“ Die Abbildung (ebenda, Tafel 8) lässt neben einem Fauteuil und einem Tabouret aus der Chambre de Parade in Herrenchiemsee einen der Stühle sowie die Palmen aus Linderhof erkennen. Die aus dem Museum ausgeliehene Stückzahl von 23 Objekten beweist, dass das Bett tatsächlich in Chicago gewesen sein muss.

56. - Bayerisches Nationalmuseum Zugangsbuch II 1889-96, S. 302.

57. - Bayerisches Nationalmuseum Zugangsbuch V 1908-1912, S. 61; Korrespondenz in den Erwerbungsakten des BNM vor 1945, Dok. 1743, König Ludwig II.-Ensemble (Bett). Kaufpreis waren 1250,- Reichsmark. 


\section{RÉSUMÉS}

König Ludwig II. von Bayern (1845-1886, regierend ab 1864) gilt als wichtiger Initiator einer hohen Blüte des Kunsthandwerks im letzten Drittel des 19. Jahrhunderts in Süddeutschland. Mit den Aufträgen für seine historistischen Schöpfungen Neuschwanstein, Linderhof und Herrenchiemsee und für weitere, orientalische und phantastische Bauten und Pläne forderte er Höchstleistungen von seinen Künstlern und Kunsthandwerkern. Einige von ihnen begründeten den Rang insbesondere Münchens als Zentrum hochrangigen Kunstgewerbes auf Jahrzehnte. Dieser Beitrag richtet sich auf die Genese des Bettes des ersten Linderhofer Schlafzimmers und seine Einbindung in die Planungsgeschichte der Schlösser Linderhof und Herrenchiemsee.

\section{INDEX}

Schlüsselwörter : Neorokoko, Neobarock, Bett Ludwig II. von Bayern, Bett Louis XIV, Chambre de Parade, Salböl Taube, Linderhof, Bildhauermöbel, Anton Pössenbacher

\section{AUTEUR}

\section{SYBE WARTENA}

Referent für Möbel, Musikinstrumente, Spiele und Stadtmodelle, Bayerisches Nationalmuseum Sybe.Wartena@bnm.mwn.de 